\title{
Supplementary data for the article:
}

Milenković, I.; Radotić, K.; Trifković, J.; Vujisić, L.; Beškoski, V. P. Screening of SemiVolatile Compounds in Plants Treated with Coated Cerium Oxide Nanoparticles by Comprehensive Two-Dimensional Gas Chromatography. Journal of Separation Science 2021, 44 (11), 2260-2268. https://doi.org/10.1002/jssc.202100145. 
Supplementary Table S1. Compounds tentatively identified by software of GCxGC-MS method in methylene chloride extracts of wheat shoot; + means presence and - means absence of compounds in the sample

\begin{tabular}{|c|c|c|c|c|c|c|c|}
\hline Compounds & Formula & $\begin{array}{c}\text { Order } \\
\text { number }\end{array}$ & RT1 & RT2 & $\begin{array}{c}\text { Similarity } \\
(\%)\end{array}$ & Presence & Treatments \\
\hline \multirow{5}{*}{$\begin{array}{l}\text { 1,2-dimethyl- } \\
\text { benzene }\end{array}$} & \multirow{5}{*}{$\mathrm{C}_{8} \mathrm{H}_{10}$} & \multirow{5}{*}{1} & \multirow{5}{*}{12.769} & \multirow{5}{*}{2.64} & \multirow{5}{*}{95} & + & Control \\
\hline & & & & & & + & $\mathrm{nCeO} 2$ \\
\hline & & & & & & + & $\mathrm{G}-\mathrm{CeO}_{2}$ \\
\hline & & & & & & + & $\mathrm{L}-\mathrm{CeO}_{2}$ \\
\hline & & & & & & + & $\mathrm{P}-\mathrm{CeO}_{2}$ \\
\hline \multirow{5}{*}{$\begin{array}{l}\text { 2,3,4-trimethyl- } \\
\text { hexane }\end{array}$} & \multirow{5}{*}{$\mathrm{C}_{9} \mathrm{H}_{20}$} & \multirow{5}{*}{2} & \multirow{5}{*}{12.948} & \multirow{5}{*}{1.35} & \multirow{5}{*}{85} & - & Control \\
\hline & & & & & & + & $\mathrm{nCeO} 2$ \\
\hline & & & & & & + & $\mathrm{G}-\mathrm{CeO}_{2}$ \\
\hline & & & & & & + & $\mathrm{L}-\mathrm{CeO}_{2}$ \\
\hline & & & & & & + & $\mathrm{P}-\mathrm{CeO}_{2}$ \\
\hline \multirow{5}{*}{$\begin{array}{l}\text { (E)-2,2-dimethyl- } \\
\text { 3-decene }\end{array}$} & \multirow{5}{*}{$\mathrm{C}_{12} \mathrm{H}_{24}$} & \multirow{5}{*}{3} & \multirow{5}{*}{13.650} & \multirow{5}{*}{1.44} & \multirow{5}{*}{85} & - & Control \\
\hline & & & & & & - & $\mathrm{nCeO} 2$ \\
\hline & & & & & & + & $\mathrm{G}-\mathrm{CeO}_{2}$ \\
\hline & & & & & & + & $\mathrm{L}-\mathrm{CeO}_{2}$ \\
\hline & & & & & & + & $\mathrm{P}-\mathrm{CeO}_{2}$ \\
\hline \multirow{5}{*}{$\begin{array}{l}\text { 2,6,6-trimethyl-1- } \\
\text { heptene }\end{array}$} & \multirow{5}{*}{$\mathrm{C}_{10} \mathrm{H}_{20}$} & & & & & + & Control \\
\hline & & & & & & + & $\mathrm{nCeO} 2$ \\
\hline & & 4 & 14.350 & 1.50 & 82 & + & $\mathrm{G}-\mathrm{CeO}_{2}$ \\
\hline & & & & & & + & $\mathrm{L}-\mathrm{CeO}_{2}$ \\
\hline & & & & & & + & $\mathrm{P}-\mathrm{CeO}_{2}$ \\
\hline & & & & & & + & Control \\
\hline & & & & & & - & $\mathrm{nCeO} 2$ \\
\hline $\begin{array}{l}\text { 3-etnyl-2-metnyl- } \\
\text { 1-hentene }\end{array}$ & $\mathrm{C}_{10} \mathrm{H}_{20}$ & 5 & 14.952 & 1.59 & 85 & + & $\mathrm{G}-\mathrm{CeO}_{2}$ \\
\hline & & & & & & + & $\mathrm{L}-\mathrm{CeO}_{2}$ \\
\hline & & & & & & - & $\mathrm{P}-\mathrm{CeO}_{2}$ \\
\hline & & & & & & + & Control \\
\hline & & & & & & + & $\mathrm{nCeO} 2$ \\
\hline $\begin{array}{l}\text { 2,4,4-trimethyl-1- } \\
\text { hexene }\end{array}$ & $\mathrm{C}_{9} \mathrm{H}_{18}$ & 6 & 15.253 & 1.65 & 86 & + & $\mathrm{G}-\mathrm{CeO}_{2}$ \\
\hline & & & & & & + & $\mathrm{L}-\mathrm{CeO}_{2}$ \\
\hline & & & & & & + & $\mathrm{P}-\mathrm{CeO}_{2}$ \\
\hline & & & & & & + & Control \\
\hline & & & & & & + & $\mathrm{nCeO} 2$ \\
\hline (E)-2-heptenal & $\mathrm{C}_{7} \mathrm{H}_{12} \mathrm{O}$ & 7 & 15.653 & 1.65 & 91 & + & $\mathrm{G}-\mathrm{CeO}_{2}$ \\
\hline & & & & & & + & $\mathrm{L}-\mathrm{CeO}_{2}$ \\
\hline & & & & & & + & $\mathrm{P}-\mathrm{CeO}_{2}$ \\
\hline & & & & & & - & Control \\
\hline & & & & & & + & $\mathrm{nCeO} 2$ \\
\hline Benzaldehyde & $\mathrm{C}_{7} \mathrm{H}_{6} \mathrm{O}$ & 8 & 15.664 & 2.31 & 85 & + & $\mathrm{G}-\mathrm{CeO}_{2}$ \\
\hline & & & & & & - & $\mathrm{L}-\mathrm{CeO}_{2}$ \\
\hline & & & & & & - & $\mathrm{P}-\mathrm{CeO}_{2}$ \\
\hline & & & & & & + & Control \\
\hline 1-methyl-2- & & & & & & + & $\mathrm{nCeO} 2$ \\
\hline propyl- & $\mathrm{C}_{10} \mathrm{H}_{20}$ & 9 & 15.953 & 1.68 & 90 & + & $\mathrm{G}-\mathrm{CeO}_{2}$ \\
\hline cyclohexane & & & & & & + & $\mathrm{L}-\mathrm{CeO}_{2}$ \\
\hline & & & & & & + & $\mathrm{P}-\mathrm{CeO}_{2}$ \\
\hline & & & & & & - & Control \\
\hline 2-methvl-nonane & $\mathrm{C}_{10} \mathrm{H}_{2}$ & 10 & 16650 & 150 & 92 & - & $\mathrm{nCeO} 2$ \\
\hline 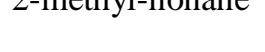 & $\mathrm{C}_{10} \mathrm{H}_{22}$ & 10 & 10.050 & 1.50 & 92 & + & $\mathrm{G}-\mathrm{CeO}_{2}$ \\
\hline & & & & & & + & $\mathrm{L}-\mathrm{CeO}_{2}$ \\
\hline
\end{tabular}




\begin{tabular}{|c|c|c|c|c|c|c|c|}
\hline Compounds & Formula & $\begin{array}{c}\text { Order } \\
\text { number }\end{array}$ & RT1 & RT2 & $\begin{array}{c}\text { Similarity } \\
(\%)\end{array}$ & Presence & Treatments \\
\hline & & & & & & + & $\mathrm{P}-\mathrm{CeO}_{2}$ \\
\hline \multirow{5}{*}{ Heptanoic acid } & \multirow{5}{*}{$\mathrm{C}_{7} \mathrm{H}_{14} \mathrm{O}_{2}$} & \multirow{5}{*}{11} & \multirow{5}{*}{16.745} & \multirow{5}{*}{1.20} & \multirow{5}{*}{84} & - & Control \\
\hline & & & & & & + & $\mathrm{nCeO} 2$ \\
\hline & & & & & & + & G-CeO ${ }_{2}$ \\
\hline & & & & & & + & $\mathrm{L}-\mathrm{CeO}_{2}$ \\
\hline & & & & & & + & $\mathrm{P}-\mathrm{CeO}_{2}$ \\
\hline \multirow{5}{*}{ 3-methyl-nonane } & \multirow{5}{*}{$\mathrm{C}_{10} \mathrm{H}_{22}$} & \multirow{5}{*}{12} & \multirow{5}{*}{16.851} & \multirow{5}{*}{1.53} & \multirow{5}{*}{90} & - & Control \\
\hline & & & & & & - & $\mathrm{nCeO} 2$ \\
\hline & & & & & & + & $\mathrm{G}-\mathrm{CeO}_{2}$ \\
\hline & & & & & & + & $\mathrm{L}-\mathrm{CeO}_{2}$ \\
\hline & & & & & & + & $\mathrm{P}-\mathrm{CeO}_{2}$ \\
\hline \multirow{5}{*}{$\begin{array}{l}(E, E)-2,4- \\
\text { heptadienal }\end{array}$} & \multirow{5}{*}{$\mathrm{C}_{7} \mathrm{H}_{10} \mathrm{O}$} & \multirow{5}{*}{13} & \multirow{5}{*}{17.054} & \multirow{5}{*}{1.74} & \multirow{5}{*}{91} & + & Control \\
\hline & & & & & & + & $\mathrm{nCeO} 2$ \\
\hline & & & & & & + & G-CeO ${ }_{2}$ \\
\hline & & & & & & + & $\mathrm{L}-\mathrm{CeO}_{2}$ \\
\hline & & & & & & + & $\mathrm{P}-\mathrm{CeO}_{2}$ \\
\hline & & & & & & - & Control \\
\hline & & & & & & + & $\mathrm{nCeO} 2$ \\
\hline $\begin{array}{l}\text { J-memyi- } \\
\text { undecane }\end{array}$ & $\mathrm{C}_{12} \mathrm{H}_{26}$ & 14 & 17.150 & 1.44 & 92 & + & G-CeO ${ }_{2}$ \\
\hline & & & & & & + & $\mathrm{L}-\mathrm{CeO}_{2}$ \\
\hline & & & & & & + & $\mathrm{P}-\mathrm{CeO}_{2}$ \\
\hline & & & & & & - & Control \\
\hline & & & & & & + & $\mathrm{nCeO} 2$ \\
\hline Octanal & $\mathrm{C}_{8} \mathrm{H}_{16} \mathrm{O}$ & 15 & 17.346 & 1.23 & 86 & + & G-CeO ${ }_{2}$ \\
\hline & & & & & & + & $\mathrm{L}-\mathrm{CeO}_{2}$ \\
\hline & & & & & & + & $\mathrm{P}-\mathrm{CeO}_{2}$ \\
\hline & & & & & & - & Control \\
\hline & & & & & & + & $\mathrm{nCeO} 2$ \\
\hline Decane & $\mathrm{C}_{10} \mathrm{H}_{22}$ & 16 & 17.852 & 1.62 & 94 & + & G-CeO ${ }_{2}$ \\
\hline & & & & & & + & $\mathrm{L}-\mathrm{CeO}_{2}$ \\
\hline & & & & & & + & $\mathrm{P}-\mathrm{CeO}_{2}$ \\
\hline & & & & & & - & Control \\
\hline & & & & & & - & $\mathrm{nCeO} 2$ \\
\hline 3,3-dimethyl- & $\mathrm{C}_{10} \mathrm{H}_{22}$ & 17 & 18.251 & 1.53 & 88 & + & G-CeO ${ }_{2}$ \\
\hline & & & & & & + & $\mathrm{L}-\mathrm{CeO}_{2}$ \\
\hline & & & & & & + & $\mathrm{P}-\mathrm{CeO}_{2}$ \\
\hline & & & & & & - & Control \\
\hline & & & & & & - & $\mathrm{nCeO} 2$ \\
\hline $\begin{array}{l}\text { 2,5,0-trimetnyl- } \\
\text { decane }\end{array}$ & $\mathrm{C}_{13} \mathrm{H}_{28}$ & 18 & 18.451 & 1.56 & 92 & + & G-CeO ${ }_{2}$ \\
\hline & & & & & & + & $\mathrm{L}-\mathrm{CeO}_{2}$ \\
\hline & & & & & & + & $\mathrm{P}-\mathrm{CeO}_{2}$ \\
\hline & & & & & & + & Control \\
\hline & & & & & & + & $\mathrm{nCeO} 2$ \\
\hline 2-ethyl-1-hexanol & $\mathrm{C}_{8} \mathrm{H}_{18} \mathrm{O}$ & 19 & 18.544 & 1.11 & 89 & + & G-CeO ${ }_{2}$ \\
\hline & & & & & & + & $\mathrm{L}-\mathrm{CeO}_{2}$ \\
\hline & & & & & & + & $\mathrm{P}-\mathrm{CeO}_{2}$ \\
\hline & & & & & & - & Control \\
\hline & & & & & & + & $\mathrm{nCeO} 2$ \\
\hline $\begin{array}{l}\text { 2,L,0-trimetnyl- } \\
\text { cyclohexanone }\end{array}$ & $\mathrm{C}_{9} \mathrm{H}_{16} \mathrm{O}$ & 20 & 18.548 & 1.38 & 86 & - & G-CeO ${ }_{2}$ \\
\hline & & & & & & - & $\mathrm{L}-\mathrm{CeO}_{2}$ \\
\hline & & & & & & - & $\mathrm{P}-\mathrm{CeO}_{2}$ \\
\hline & & & & & & - & Control \\
\hline $\begin{array}{l}\text { 2,o, I-trimethyl- } \\
\text { decane }\end{array}$ & $\mathrm{C}_{13} \mathrm{H}_{28}$ & 21 & 18.651 & 1.53 & 92 & - & $\mathrm{nCeO} 2$ \\
\hline & & & & & & + & G-CeO ${ }_{2}$ \\
\hline
\end{tabular}




\begin{tabular}{|c|c|c|c|c|c|c|c|}
\hline Compounds & Formula & $\begin{array}{c}\text { Order } \\
\text { number }\end{array}$ & RT1 & RT2 & $\begin{array}{c}\text { Similarity } \\
(\%)\end{array}$ & Presence & Treatments \\
\hline & & & & & & + & $\mathrm{L}-\mathrm{CeO}_{2}$ \\
\hline & & & & & & - & $\mathrm{P}-\mathrm{CeO}_{2}$ \\
\hline \multirow{5}{*}{$\begin{array}{l}\text { (S)-1-methyl-4-(1- } \\
\text { methylethenyl)- } \\
\text { cyclohexene }\end{array}$} & \multirow{5}{*}{$\mathrm{C}_{10} \mathrm{H}_{16}$} & \multirow{5}{*}{22} & \multirow{5}{*}{18.667} & \multirow{5}{*}{2.40} & \multirow{5}{*}{86} & + & Control \\
\hline & & & & & & - & $\mathrm{nCeO} 2$ \\
\hline & & & & & & - & $\mathrm{G}-\mathrm{CeO}_{2}$ \\
\hline & & & & & & + & $\mathrm{L}-\mathrm{CeO}_{2}$ \\
\hline & & & & & & + & $\mathrm{P}-\mathrm{CeO}_{2}$ \\
\hline \multirow{5}{*}{ 4-methyl-decane } & \multirow{5}{*}{$\mathrm{C}_{11} \mathrm{H}_{24}$} & \multirow{5}{*}{23} & \multirow{5}{*}{18.751} & \multirow{5}{*}{1.53} & \multirow{5}{*}{92} & - & Control \\
\hline & & & & & & - & $\mathrm{nCeO} 2$ \\
\hline & & & & & & + & $\mathrm{G}-\mathrm{CeO}_{2}$ \\
\hline & & & & & & + & $\mathrm{L}-\mathrm{CeO}_{2}$ \\
\hline & & & & & & - & $\mathrm{P}-\mathrm{CeO}_{2}$ \\
\hline \multirow{5}{*}{ 1-phenyl-ethanone } & \multirow{5}{*}{$\mathrm{C}_{8} \mathrm{H}_{8} \mathrm{O}$} & \multirow{5}{*}{24} & \multirow{5}{*}{19.363} & \multirow{5}{*}{2.28} & & - & Control \\
\hline & & & & & & - & $\mathrm{nCeO} 2$ \\
\hline & & & & & 86 & + & $\mathrm{G}-\mathrm{CeO}_{2}$ \\
\hline & & & & & & + & $\mathrm{L}-\mathrm{CeO}_{2}$ \\
\hline & & & & & & + & $\mathrm{P}-\mathrm{CeO}_{2}$ \\
\hline & & & & & & + & Control \\
\hline & & & & & & + & $\mathrm{nCeO} 2$ \\
\hline henzaldehyde & $\mathrm{C}_{8} \mathrm{H}_{8} \mathrm{O}$ & 25 & 19.460 & 2.10 & 89 & + & $\mathrm{G}-\mathrm{CeO}_{2}$ \\
\hline & & & & & & + & $\mathrm{L}-\mathrm{CeO}_{2}$ \\
\hline & & & & & & + & $\mathrm{P}-\mathrm{CeO}_{2}$ \\
\hline & & & & & & + & Control \\
\hline & & & & & & + & $\mathrm{nCeO} 2$ \\
\hline $\begin{array}{l}\text { 4-methyl- } \\
\text { benzaldehyde }\end{array}$ & $\mathrm{C}_{8} \mathrm{H}_{8} \mathrm{O}$ & 26 & 19.861 & 2.13 & 96 & + & $\mathrm{G}-\mathrm{CeO}_{2}$ \\
\hline & & & & & & + & $\mathrm{L}-\mathrm{CeO}_{2}$ \\
\hline & & & & & & + & $\mathrm{P}-\mathrm{CeO}_{2}$ \\
\hline & & & & & & - & Control \\
\hline & & & & & & + & $\mathrm{nCeO} 2$ \\
\hline $\begin{array}{l}\text { 4,0-armetny-- } \\
\text { dodecane }\end{array}$ & $\mathrm{C}_{14} \mathrm{H}_{30}$ & 27 & 19.951 & 1.56 & 93 & + & G-CeO ${ }_{2}$ \\
\hline & & & & & & + & $\mathrm{L}-\mathrm{CeO}_{2}$ \\
\hline & & & & & & + & $\mathrm{P}-\mathrm{CeO}_{2}$ \\
\hline & & & & & & + & Control \\
\hline & & & & & & + & $\mathrm{nCeO} 2$ \\
\hline Cyclohexanol & $\mathrm{C}_{6} \mathrm{H}_{12} \mathrm{O}$ & 28 & 20.144 & 1.08 & 83 & + & G-CeO ${ }_{2}$ \\
\hline & & & & & & + & $\mathrm{L}-\mathrm{CeO}_{2}$ \\
\hline & & & & & & + & $\mathrm{P}-\mathrm{CeO}_{2}$ \\
\hline & & & & & & - & Control \\
\hline 1-methyl-4-(1- & & & & & & + & $\mathrm{nCeO} 2$ \\
\hline methylethyl)- & $\mathrm{C}_{10} \mathrm{H}_{14}$ & 29 & 20.446 & 1.26 & 80 & + & G-CeO ${ }_{2}$ \\
\hline benzene & & & & & & - & $\mathrm{L}-\mathrm{CeO}_{2}$ \\
\hline & & & & & & - & $\mathrm{P}-\mathrm{CeO}_{2}$ \\
\hline & & & & & & - & Control \\
\hline & & & & & & - & $\mathrm{nCeO} 2$ \\
\hline 1-decanol & $\mathrm{C}_{10} \mathrm{H}_{22} \mathrm{O}$ & 30 & 20.554 & 1.68 & 84 & - & G-CeO ${ }_{2}$ \\
\hline & & & & & & + & $\mathrm{L}-\mathrm{CeO}_{2}$ \\
\hline & & & & & & + & $\mathrm{P}-\mathrm{CeO}_{2}$ \\
\hline & & & & & & + & Control \\
\hline & & & & & & + & $\mathrm{nCeO} 2$ \\
\hline Nonanal & $\mathrm{C}_{9} \mathrm{H}_{18} \mathrm{O}$ & 31 & 20.845 & 1.17 & 95 & + & G-CeO ${ }_{2}$ \\
\hline & & & & & & + & $\mathrm{L}-\mathrm{CeO}_{2}$ \\
\hline & & & & & & + & $\mathrm{P}-\mathrm{CeO}_{2}$ \\
\hline & & & & & & - & Control \\
\hline 2,6-dımethyl- & $\mathrm{C}_{8} \mathrm{H}_{16} \mathrm{O}$ & 32 & 20.952 & 1.59 & 86 & + & $\mathrm{nCeO} 2$ \\
\hline & & & & & & + & $\mathrm{G}-\mathrm{CeO}_{2}$ \\
\hline
\end{tabular}




\begin{tabular}{|c|c|c|c|c|c|c|c|}
\hline Compounds & Formula & $\begin{array}{c}\text { Order } \\
\text { number }\end{array}$ & RT1 & RT2 & $\begin{array}{c}\text { Similarity } \\
(\%)\end{array}$ & Presence & Treatments \\
\hline & & & & & & + & $\mathrm{L}-\mathrm{CeO}_{2}$ \\
\hline & & & & & & - & $\mathrm{P}-\mathrm{CeO}_{2}$ \\
\hline \multirow{5}{*}{$\begin{array}{l}\text { 4,5-dimethyl- } \\
\text { nonane }\end{array}$} & \multirow{5}{*}{$\mathrm{C}_{11} \mathrm{H}_{24}$} & \multirow{5}{*}{33} & \multirow{5}{*}{21.352} & \multirow{5}{*}{1.62} & \multirow{5}{*}{93} & - & Control \\
\hline & & & & & & - & $\mathrm{nCeO} 2$ \\
\hline & & & & & & + & $\mathrm{G}-\mathrm{CeO}_{2}$ \\
\hline & & & & & & + & $\mathrm{L}-\mathrm{CeO}_{2}$ \\
\hline & & & & & & + & $\mathrm{P}-\mathrm{CeO}_{2}$ \\
\hline \multirow{5}{*}{$\begin{array}{l}\text { 2-ethyl-hexanoic } \\
\text { acid }\end{array}$} & \multirow{5}{*}{$\mathrm{C}_{8} \mathrm{H}_{16} \mathrm{O}_{2}$} & \multirow{5}{*}{34} & \multirow{5}{*}{21.443} & \multirow{5}{*}{1.08} & \multirow{5}{*}{82} & + & Control \\
\hline & & & & & & + & $\mathrm{nCeO} 2$ \\
\hline & & & & & & + & $\mathrm{G}-\mathrm{CeO}_{2}$ \\
\hline & & & & & & + & $\mathrm{L}-\mathrm{CeO}_{2}$ \\
\hline & & & & & & + & $\mathrm{P}-\mathrm{CeO}_{2}$ \\
\hline \multirow{5}{*}{$\begin{array}{c}\text { 2,6,11-trimethyl- } \\
\text { dodecane }\end{array}$} & \multirow{5}{*}{$\mathrm{C}_{15} \mathrm{H}_{32}$} & \multirow{5}{*}{35} & \multirow{5}{*}{21.552} & \multirow{5}{*}{1.59} & & - & Control \\
\hline & & & & & & - & $\mathrm{nCeO} 2$ \\
\hline & & & & & 91 & + & $\mathrm{G}-\mathrm{CeO} 2$ \\
\hline & & & & & & + & $\mathrm{L}-\mathrm{CeO}_{2}$ \\
\hline & & & & & & + & $\mathrm{P}-\mathrm{CeO}_{2}$ \\
\hline & & & & & & - & Control \\
\hline $1,2,3,4-$ & & & & & & + & $\mathrm{nCeO} 2$ \\
\hline tetramethyl- & $\mathrm{C}_{10} \mathrm{H}_{14}$ & 36 & 21.647 & 1.32 & 91 & + & $\mathrm{G}-\mathrm{CeO}_{2}$ \\
\hline & & & & & & - & $\mathrm{L}-\mathrm{CeO}_{2}$ \\
\hline & & & & & & - & $\mathrm{P}-\mathrm{CeO}_{2}$ \\
\hline & & & & & & - & Control \\
\hline & & & & & & - & $\mathrm{nCeO} 2$ \\
\hline Dodecane & $\mathrm{C}_{12} \mathrm{H}_{26}$ & 37 & 21.752 & 1.62 & 87 & - & $\mathrm{G}-\mathrm{CeO}_{2}$ \\
\hline & & & & & & + & $\mathrm{L}-\mathrm{CeO}_{2}$ \\
\hline & & & & & & + & $\mathrm{P}-\mathrm{CeO}_{2}$ \\
\hline & & & & & & - & Control \\
\hline & & & & & & - & $\mathrm{nCeO} 2$ \\
\hline Hexadecane & $\mathrm{C}_{16} \mathrm{H}_{34}$ & 38 & 22.153 & 1.65 & 85 & - & G-CeO ${ }_{2}$ \\
\hline & & & & & & + & $\mathrm{L}-\mathrm{CeO}_{2}$ \\
\hline & & & & & & + & $\mathrm{P}-\mathrm{CeO}_{2}$ \\
\hline & & & & & & - & Control \\
\hline & & & & & & + & $\mathrm{nCeO} 2$ \\
\hline 1-phenyl-1- & $\mathrm{C}_{9} \mathrm{H}_{10} \mathrm{O}$ & 39 & 22.559 & 2.04 & 85 & + & G-CeO ${ }_{2}$ \\
\hline & & & & & & + & $\mathrm{L}-\mathrm{CeO}_{2}$ \\
\hline & & & & & & + & $\mathrm{P}-\mathrm{CeO}_{2}$ \\
\hline & & & & & & - & Control \\
\hline & & & & & & + & $\mathrm{nCeO} 2$ \\
\hline Octanoic acid & $\mathrm{C}_{8} \mathrm{H}_{16} \mathrm{O}_{2}$ & 40 & 22.945 & 1.20 & 88 & + & $\mathrm{G}-\mathrm{CeO}_{2}$ \\
\hline & & & & & & + & $\mathrm{L}-\mathrm{CeO}_{2}$ \\
\hline & & & & & & + & $\mathrm{P}-\mathrm{CeO}_{2}$ \\
\hline & & & & & & + & Control \\
\hline & & & & & & + & $\mathrm{nCeO} 2$ \\
\hline $\begin{array}{l}\text { 3,J-dimetnyl- } \\
\text { henzaldehyde }\end{array}$ & $\mathrm{C}_{9} \mathrm{H}_{10} \mathrm{O}$ & 41 & 22.958 & 1.92 & 81 & + & G-CeO ${ }_{2}$ \\
\hline & & & & & & + & $\mathrm{L}-\mathrm{CeO}_{2}$ \\
\hline & & & & & & - & $\mathrm{P}-\mathrm{CeO}_{2}$ \\
\hline & & & & & & - & Control \\
\hline & & & & & & + & $\mathrm{nCeO} 2$ \\
\hline methylphenyl)- & $\mathrm{C}_{9} \mathrm{H}_{10} \mathrm{O}$ & 42 & 23.160 & 2.10 & 94 & + & G-CeO ${ }_{2}$ \\
\hline ethanone & & & & & & + & $\mathrm{L}-\mathrm{CeO}_{2}$ \\
\hline & & & & & & + & $\mathrm{P}-\mathrm{CeO}_{2}$ \\
\hline & & & & & & - & Control \\
\hline $\begin{array}{l}\text { 2-methyl- } \\
\text { undecane }\end{array}$ & $\mathrm{C}_{12} \mathrm{H}_{26}$ & 43 & 23.254 & 1.71 & 91 & - & $\mathrm{nCeO} 2$ \\
\hline & & & & & & - & $\mathrm{G}-\mathrm{CeO}_{2}$ \\
\hline
\end{tabular}




\begin{tabular}{|c|c|c|c|c|c|c|c|}
\hline Compounds & Formula & $\begin{array}{c}\text { Order } \\
\text { number }\end{array}$ & RT1 & RT2 & $\begin{array}{c}\text { Similarity } \\
(\%)\end{array}$ & Presence & Treatments \\
\hline & & & & & & + & $\mathrm{L}-\mathrm{CeO}_{2}$ \\
\hline & & & & & & + & $\mathrm{P}-\mathrm{CeO}_{2}$ \\
\hline \multirow{5}{*}{ Naphthalene } & \multirow{5}{*}{$\mathrm{C}_{10} \mathrm{H}_{8}$} & \multirow{5}{*}{44} & \multirow{5}{*}{23.460} & \multirow{5}{*}{2.10} & \multirow{5}{*}{95} & + & Control \\
\hline & & & & & & + & $\mathrm{nCeO} 2$ \\
\hline & & & & & & + & $\mathrm{G}-\mathrm{CeO}_{2}$ \\
\hline & & & & & & + & $\mathrm{L}-\mathrm{CeO}_{2}$ \\
\hline & & & & & & + & $\mathrm{P}-\mathrm{CeO}_{2}$ \\
\hline \multirow{5}{*}{ Tetradecane } & \multirow{5}{*}{$\mathrm{C}_{14} \mathrm{H}_{30}$} & \multirow{5}{*}{45} & \multirow{5}{*}{23.653} & \multirow{5}{*}{1.65} & \multirow{5}{*}{86} & - & Control \\
\hline & & & & & & - & $\mathrm{nCeO} 2$ \\
\hline & & & & & & - & $\mathrm{G}-\mathrm{CeO}_{2}$ \\
\hline & & & & & & + & $\mathrm{L}-\mathrm{CeO}_{2}$ \\
\hline & & & & & & + & $\mathrm{P}-\mathrm{CeO}_{2}$ \\
\hline \multirow{5}{*}{ Decanal } & \multirow{5}{*}{$\mathrm{C}_{10} \mathrm{H}_{20} \mathrm{O}$} & \multirow{5}{*}{46} & \multirow{5}{*}{23.945} & \multirow{5}{*}{1.17} & & + & Control \\
\hline & & & & & & + & $\mathrm{nCeO} 2$ \\
\hline & & & & & 95 & + & $\mathrm{G}-\mathrm{CeO}_{2}$ \\
\hline & & & & & & + & $\mathrm{L}-\mathrm{CeO}_{2}$ \\
\hline & & & & & & + & $\mathrm{P}-\mathrm{CeO}_{2}$ \\
\hline & & & & & & - & Control \\
\hline $1,2,3,4,5-$ & & & & & & + & $\mathrm{nCeO} 2$ \\
\hline pentamethyl- & $\mathrm{C}_{11} \mathrm{H}_{16}$ & 47 & 24.047 & 1.32 & 88 & + & $\mathrm{G}-\mathrm{CeO}_{2}$ \\
\hline benzene & & & & & & + & $\mathrm{L}-\mathrm{CeO}_{2}$ \\
\hline & & & & & & - & $\mathrm{P}-\mathrm{CeO}_{2}$ \\
\hline & & & & & & + & Control \\
\hline & & & & & & + & $\mathrm{nCeO} 2$ \\
\hline benzaldehyde & $\mathrm{C}_{9} \mathrm{H}_{10} \mathrm{O}$ & 48 & 24.160 & 2.13 & 96 & + & $\mathrm{G}-\mathrm{CeO}_{2}$ \\
\hline & & & & & & + & $\mathrm{L}-\mathrm{CeO}_{2}$ \\
\hline & & & & & & + & $\mathrm{P}-\mathrm{CeO}_{2}$ \\
\hline & & & & & & + & Control \\
\hline & & & & & & + & $\mathrm{nCeO} 2$ \\
\hline 2-phenoxy- & $\mathrm{C}_{8} \mathrm{H}_{10} \mathrm{O}_{2}$ & 49 & 24.165 & 2.37 & 92 & + & G-CeO ${ }_{2}$ \\
\hline & & & & & & + & $\mathrm{L}-\mathrm{CeO}_{2}$ \\
\hline & & & & & & + & $\mathrm{P}-\mathrm{CeO}_{2}$ \\
\hline & & & & & & + & Control \\
\hline & & & & & & + & $\mathrm{nCeO} 2$ \\
\hline Benzothiazole & $\mathrm{C}_{7} \mathrm{H}_{5} \mathrm{NS}$ & 50 & 24.374 & 2.88 & 91 & + & G-CeO ${ }_{2}$ \\
\hline & & & & & & + & $\mathrm{L}-\mathrm{CeO}_{2}$ \\
\hline & & & & & & + & $\mathrm{P}-\mathrm{CeO}_{2}$ \\
\hline & & & & & & + & Control \\
\hline 2,6,6-trimethyl-1- & & & & & & + & $\mathrm{nCeO} 2$ \\
\hline cyclohexene-1- & $\mathrm{C}_{10} \mathrm{H}_{16} \mathrm{O}$ & 51 & 24.453 & 1.65 & 91 & + & G-CeO ${ }_{2}$ \\
\hline carboxaldehyde & & & & & & + & $\mathrm{L}-\mathrm{CeO}_{2}$ \\
\hline & & & & & & + & $\mathrm{P}-\mathrm{CeO}_{2}$ \\
\hline & & & & & & + & Control \\
\hline & & & & & & + & $\mathrm{nCeO} 2$ \\
\hline$(E)$-2-decenal & $\mathrm{C}_{10} \mathrm{H}_{18} \mathrm{O}$ & 52 & 25.547 & 1.32 & 88 & + & G-CeO ${ }_{2}$ \\
\hline & & & & & & + & $\mathrm{L}-\mathrm{CeO}_{2}$ \\
\hline & & & & & & + & $\mathrm{P}-\mathrm{CeO}_{2}$ \\
\hline & & & & & & - & Control \\
\hline & & & & & & - & $\mathrm{nCeO} 2$ \\
\hline $\begin{array}{l}\alpha, \alpha \text { - armetnyl- } \\
\text { henzenemethanol }\end{array}$ & $\mathrm{C}_{9} \mathrm{H}_{12} \mathrm{O}$ & 53 & 25.562 & 2.19 & 80 & + & G-CeO ${ }_{2}$ \\
\hline & & & & & & - & $\mathrm{L}-\mathrm{CeO}_{2}$ \\
\hline & & & & & & - & $\mathrm{P}-\mathrm{CeO}_{2}$ \\
\hline 2,6,6-trimethyl-1- & & & & & & + & Control \\
\hline cyclohexene-1- & $\mathrm{C}_{11} \mathrm{H}_{18} \mathrm{O}$ & 54 & 25.650 & 1.50 & 94 & + & $\mathrm{nCeO} 2$ \\
\hline acetaldehyde & & & & & & + & $\mathrm{G}-\mathrm{CeO}_{2}$ \\
\hline
\end{tabular}




\begin{tabular}{|c|c|c|c|c|c|c|c|}
\hline Compounds & Formula & $\begin{array}{c}\text { Order } \\
\text { number }\end{array}$ & RT1 & RT2 & $\begin{array}{c}\text { Similarity } \\
(\%)\end{array}$ & Presence & Treatments \\
\hline & & & & & & + & $\mathrm{L}-\mathrm{CeO}_{2}$ \\
\hline & & & & & & + & $\mathrm{P}-\mathrm{CeO}_{2}$ \\
\hline \multirow{5}{*}{ Nonanoic acid } & \multirow{5}{*}{$\mathrm{C}_{9} \mathrm{H}_{18} \mathrm{O}_{2}$} & \multirow{5}{*}{55} & \multirow{5}{*}{25.746} & \multirow{5}{*}{1.23} & \multirow{5}{*}{91} & + & Control \\
\hline & & & & & & + & $\mathrm{nCeO} 2$ \\
\hline & & & & & & + & $\mathrm{G}-\mathrm{CeO}_{2}$ \\
\hline & & & & & & + & $\mathrm{L}-\mathrm{CeO}_{2}$ \\
\hline & & & & & & + & $\mathrm{P}-\mathrm{CeO}_{2}$ \\
\hline \multirow{5}{*}{$\begin{array}{c}\text { Methyl-1,2- } \\
\text { benzenecarboxylate }\end{array}$} & \multirow{5}{*}{$\mathrm{C}_{9} \mathrm{H}_{8} \mathrm{O}_{4}$} & \multirow{5}{*}{56} & \multirow{5}{*}{26.380} & \multirow{5}{*}{3.30} & \multirow{5}{*}{92} & + & Control \\
\hline & & & & & & + & $\mathrm{nCeO} 2$ \\
\hline & & & & & & + & $\mathrm{G}-\mathrm{CeO}_{2}$ \\
\hline & & & & & & + & $\mathrm{L}-\mathrm{CeO}_{2}$ \\
\hline & & & & & & + & $\mathrm{P}-\mathrm{CeO}_{2}$ \\
\hline \multirow{5}{*}{$\begin{array}{c}\text { 3-(1,1- } \\
\text { dimethylethyl)- } \\
\text { phenol }\end{array}$} & \multirow{5}{*}{$\mathrm{C}_{10} \mathrm{H}_{16} \mathrm{O}$} & \multirow{5}{*}{57} & \multirow{5}{*}{26.455} & \multirow{5}{*}{1.80} & & + & Control \\
\hline & & & & & & + & $\mathrm{nCeO} 2$ \\
\hline & & & & & 91 & + & $\mathrm{G}-\mathrm{CeO}_{2}$ \\
\hline & & & & & & + & $\mathrm{L}-\mathrm{CeO}_{2}$ \\
\hline & & & & & & + & $\mathrm{P}-\mathrm{CeO}_{2}$ \\
\hline & & & & & & - & Control \\
\hline 2,3,5,8- & & & & & & + & $\mathrm{nCeO} 2$ \\
\hline tetramethyl- & $\mathrm{C}_{14} \mathrm{H}_{30}$ & 58 & 26.755 & 1.74 & 81 & + & $\mathrm{G}-\mathrm{CeO}_{2}$ \\
\hline & & & & & & + & $\mathrm{L}-\mathrm{CeO}_{2}$ \\
\hline & & & & & & + & $\mathrm{P}-\mathrm{CeO}_{2}$ \\
\hline & & & & & & - & Control \\
\hline & & & & & & + & $\mathrm{nCeO} 2$ \\
\hline 1-metnyı- & $\mathrm{C}_{11} \mathrm{H}_{10}$ & 59 & 26.759 & 2.04 & 89 & + & $\mathrm{G}-\mathrm{CeO} 2$ \\
\hline & & & & & & + & $\mathrm{L}-\mathrm{CeO}_{2}$ \\
\hline & & & & & & + & $\mathrm{P}-\mathrm{CeO}_{2}$ \\
\hline & & & & & & + & Control \\
\hline & & & & & & + & $\mathrm{nCeO} 2$ \\
\hline Undecanal & $\mathrm{C}_{11} \mathrm{H}_{22} \mathrm{O}$ & 60 & 26.945 & 1.20 & 92 & + & G-CeO ${ }_{2}$ \\
\hline & & & & & & + & $\mathrm{L}-\mathrm{CeO}_{2}$ \\
\hline & & & & & & + & $\mathrm{P}-\mathrm{CeO}_{2}$ \\
\hline & & & & & & - & Control \\
\hline & & & & & & + & $\mathrm{nCeO} 2$ \\
\hline$(E, E)-2,4-$ & $\mathrm{C}_{10} \mathrm{H}_{16} \mathrm{O}$ & 61 & 27.051 & 1.53 & 87 & + & G-CeO ${ }_{2}$ \\
\hline & & & & & & + & $\mathrm{L}-\mathrm{CeO}_{2}$ \\
\hline & & & & & & + & $\mathrm{P}-\mathrm{CeO}_{2}$ \\
\hline & & & & & & - & Control \\
\hline & & & & & & + & $\mathrm{nCeO} 2$ \\
\hline 2-methyl- & $\mathrm{C}_{11} \mathrm{H}_{10}$ & 62 & 27.162 & 2.19 & 83 & + & G-CeO ${ }_{2}$ \\
\hline & & & & & & + & $\mathrm{L}-\mathrm{CeO}_{2}$ \\
\hline & & & & & & + & $\mathrm{P}-\mathrm{CeO}_{2}$ \\
\hline & & & & & & + & Control \\
\hline & & & & & & + & $\mathrm{nCeO} 2$ \\
\hline $\begin{array}{c}1,2,3 \text {-propanetriol } \\
\text { triacetate }\end{array}$ & $\mathrm{C}_{9} \mathrm{H}_{14} \mathrm{O}_{6}$ & 63 & 27.459 & 1.98 & 95 & + & G-CeO ${ }_{2}$ \\
\hline & & & & & & + & $\mathrm{L}-\mathrm{CeO}_{2}$ \\
\hline & & & & & & + & $\mathrm{P}-\mathrm{CeO}_{2}$ \\
\hline & & & & & & + & Control \\
\hline & & & & & & + & $\mathrm{nCeO} 2$ \\
\hline 2-dodecenal & $\mathrm{C}_{12} \mathrm{H}_{22} \mathrm{O}$ & 64 & 28.448 & 1.35 & 87 & + & G-CeO ${ }_{2}$ \\
\hline & & & & & & + & $\mathrm{L}-\mathrm{CeO}_{2}$ \\
\hline & & & & & & + & $\mathrm{P}-\mathrm{CeO}_{2}$ \\
\hline 1,2-dihydro-1,1,6- & & & & & & + & Control \\
\hline trimethyl- & $\mathrm{C}_{13} \mathrm{H}_{16}$ & 65 & 28.453 & 1.65 & 88 & + & $\mathrm{nCeO} 2$ \\
\hline naphthalene & & & & & & + & $\mathrm{G}-\mathrm{CeO}_{2}$ \\
\hline
\end{tabular}




\begin{tabular}{|c|c|c|c|c|c|c|c|}
\hline Compounds & Formula & $\begin{array}{c}\text { Order } \\
\text { number }\end{array}$ & RT1 & RT2 & $\begin{array}{c}\text { Similarity } \\
(\%)\end{array}$ & Presence & Treatments \\
\hline & & & & & & + & $\mathrm{L}-\mathrm{CeO}_{2}$ \\
\hline & & & & & & + & $\mathrm{P}-\mathrm{CeO}_{2}$ \\
\hline \multirow{5}{*}{$\begin{array}{c}1,2,3,4- \\
\text { tetrahydro-1,1,6- } \\
\text { trimethyl- } \\
\text { naphthalene }\end{array}$} & \multirow{5}{*}{$\mathrm{C}_{13} \mathrm{H}_{18}$} & \multirow{5}{*}{66} & \multirow{5}{*}{28.551} & \multirow{5}{*}{1.56} & \multirow{5}{*}{94} & + & Control \\
\hline & & & & & & + & $\mathrm{nCeO} 2$ \\
\hline & & & & & & + & G-CeO ${ }_{2}$ \\
\hline & & & & & & + & $\mathrm{L}-\mathrm{CeO}_{2}$ \\
\hline & & & & & & + & $\mathrm{P}-\mathrm{CeO}_{2}$ \\
\hline \multirow{5}{*}{$\begin{array}{l}\text { 4-hydroxy-3- } \\
\text { metoxybenzaldeh } \\
\text { yde }\end{array}$} & \multirow{5}{*}{$\mathrm{C}_{8} \mathrm{H}_{8} \mathrm{O}_{3}$} & \multirow{5}{*}{67} & \multirow{5}{*}{28.875} & \multirow{5}{*}{2.97} & \multirow{5}{*}{85} & - & Control \\
\hline & & & & & & - & $\mathrm{nCeO} 2$ \\
\hline & & & & & & + & $\mathrm{G}-\mathrm{CeO}_{2}$ \\
\hline & & & & & & + & $\mathrm{L}-\mathrm{CeO}_{2}$ \\
\hline & & & & & & - & $\mathrm{P}-\mathrm{CeO}_{2}$ \\
\hline \multirow{5}{*}{$\begin{array}{l}\text { 2-ethenyl- } \\
\text { naphthalene }\end{array}$} & \multirow{5}{*}{$\mathrm{C}_{12} \mathrm{H}_{10}$} & \multirow{5}{*}{68} & \multirow{5}{*}{28.962} & \multirow{5}{*}{2.19} & & + & Control \\
\hline & & & & & & + & $\mathrm{nCeO} 2$ \\
\hline & & & & & 90 & + & $\mathrm{G}-\mathrm{CeO}_{2}$ \\
\hline & & & & & & + & $\mathrm{L}-\mathrm{CeO}_{2}$ \\
\hline & & & & & & + & $\mathrm{P}-\mathrm{CeO}_{2}$ \\
\hline & & & & & & + & Control \\
\hline & & & & & & + & $\mathrm{nCeO} 2$ \\
\hline Dodecanal & $\mathrm{C}_{12} \mathrm{H}_{24} \mathrm{O}$ & 69 & 29.646 & 1.23 & 96 & + & $\mathrm{G}-\mathrm{CeO}_{2}$ \\
\hline & & & & & & + & $\mathrm{L}-\mathrm{CeO}_{2}$ \\
\hline & & & & & & + & $\mathrm{P}-\mathrm{CeO}_{2}$ \\
\hline & & & & & & + & Control \\
\hline & & & & & & + & $\mathrm{nCeO} 2$ \\
\hline naphthalene & $\mathrm{C}_{12} \mathrm{H}_{12}$ & 70 & 29.759 & 1.98 & 95 & + & $\mathrm{G}-\mathrm{CeO}_{2}$ \\
\hline & & & & & & + & $\mathrm{L}-\mathrm{CeO}_{2}$ \\
\hline & & & & & & + & $\mathrm{P}-\mathrm{CeO}_{2}$ \\
\hline & & & & & & + & Control \\
\hline & & & & & & + & $\mathrm{nCeO} 2$ \\
\hline $\begin{array}{c}1,8 \text {-dmmetnyl- } \\
\text { naphthalene }\end{array}$ & $\mathrm{C}_{12} \mathrm{H}_{12}$ & 71 & 30.160 & 2.07 & 86 & + & G-CeO ${ }_{2}$ \\
\hline & & & & & & + & $\mathrm{L}-\mathrm{CeO}_{2}$ \\
\hline & & & & & & + & $\mathrm{P}-\mathrm{CeO}_{2}$ \\
\hline$(1 R, 2 S, 7 S, 9 S)-$ & & & & & & - & Control \\
\hline 3,3,7-trimethyl-8- & & & & & & + & $\mathrm{nCeO} 2$ \\
\hline methylidenetricycl & $\mathrm{C}_{15} \mathrm{H}_{24}$ & 72 & 30.347 & 1.26 & 92 & + & $\mathrm{G}-\mathrm{CeO}_{2}$ \\
\hline $\mathrm{o}\left[5.4 .0 .0^{2,9}\right]$ undec & & & & & & + & $\mathrm{L}-\mathrm{CeO}_{2}$ \\
\hline an & & & & & & + & $\mathrm{P}-\mathrm{CeO}_{2}$ \\
\hline & & & & & & - & Control \\
\hline $1-(2,4-$ & & & & & & + & $\mathrm{nCeO} 2$ \\
\hline dimethylphenyl)- & $\mathrm{C}_{11} \mathrm{H}_{14} \mathrm{O}$ & 73 & 30.374 & 2.88 & 81 & + & G-CeO ${ }_{2}$ \\
\hline 1-propanone & & & & & & + & $\mathrm{L}-\mathrm{CeO}_{2}$ \\
\hline & & & & & & + & $\mathrm{P}-\mathrm{CeO}_{2}$ \\
\hline & & & & & & + & Control \\
\hline 6,10-dimethyl- & & & & & & + & $\mathrm{nCeO} 2$ \\
\hline 5,9-undecadien-2- & $\mathrm{C}_{13} \mathrm{H}_{22} \mathrm{O}$ & 74 & 30.749 & 1.41 & 89 & + & G-CeO ${ }_{2}$ \\
\hline & & & & & & + & $\mathrm{L}-\mathrm{CeO}_{2}$ \\
\hline & & & & & & + & $\mathrm{P}-\mathrm{CeO}_{2}$ \\
\hline & & & & & & - & Control \\
\hline & & & & & & + & $\mathrm{nCeO} 2$ \\
\hline 1-hexadecanol & $\mathrm{C}_{16} \mathrm{H}_{34} \mathrm{O}$ & 75 & 31.142 & 0.96 & 88 & - & G-CeO ${ }_{2}$ \\
\hline & & & & & & + & $\mathrm{L}-\mathrm{CeO}_{2}$ \\
\hline & & & & & & + & $\mathrm{P}-\mathrm{CeO}_{2}$ \\
\hline & & & & & & + & Control \\
\hline 1-dodecanol & $\mathrm{C}_{12} \mathrm{H}_{26} \mathrm{O}$ & 76 & 31.645 & 1.17 & 96 & + & $\mathrm{nCeO} 2$ \\
\hline & & & & & & + & $\mathrm{G}-\mathrm{CeO}_{2}$ \\
\hline
\end{tabular}




\begin{tabular}{|c|c|c|c|c|c|c|c|}
\hline Compounds & Formula & $\begin{array}{c}\text { Order } \\
\text { number }\end{array}$ & RT1 & RT2 & $\begin{array}{c}\text { Similarity } \\
(\%)\end{array}$ & Presence & Treatments \\
\hline & & & & & & + & $\mathrm{L}-\mathrm{CeO}_{2}$ \\
\hline & & & & & & + & $\mathrm{P}-\mathrm{CeO}_{2}$ \\
\hline \multirow{5}{*}{$\begin{array}{l}\text { 4-(2,6,6-trimethyl- } \\
\text { 1-cyclohexen-2- } \\
\text { yl)-3-buten-2-one }\end{array}$} & \multirow{5}{*}{$\mathrm{C}_{13} \mathrm{H}_{20} \mathrm{O}$} & \multirow{5}{*}{77} & \multirow{5}{*}{31.753} & \multirow{5}{*}{1.62} & \multirow{5}{*}{93} & + & Control \\
\hline & & & & & & + & $\mathrm{nCeO} 2$ \\
\hline & & & & & & + & $\mathrm{G}-\mathrm{CeO}_{2}$ \\
\hline & & & & & & + & $\mathrm{L}-\mathrm{CeO}_{2}$ \\
\hline & & & & & & + & $\mathrm{P}-\mathrm{CeO}_{2}$ \\
\hline \multirow{5}{*}{$\begin{array}{l}\text { 10-methyl-8- } \\
\text { tetradecenyl } \\
\text { acetate }\end{array}$} & \multirow{5}{*}{$\mathrm{C}_{17} \mathrm{H}_{32} \mathrm{O}_{2}$} & \multirow{5}{*}{78} & \multirow{5}{*}{31.967} & \multirow{5}{*}{2.46} & \multirow{5}{*}{81} & + & Control \\
\hline & & & & & & + & $\mathrm{nCeO} 2$ \\
\hline & & & & & & + & $\mathrm{G}-\mathrm{CeO}_{2}$ \\
\hline & & & & & & + & $\mathrm{L}-\mathrm{CeO}_{2}$ \\
\hline & & & & & & - & $\mathrm{P}-\mathrm{CeO}_{2}$ \\
\hline \multirow{5}{*}{ Tridecanal } & \multirow{5}{*}{$\mathrm{C}_{13} \mathrm{H}_{26} \mathrm{O}$} & \multirow{5}{*}{79} & \multirow{5}{*}{32.246} & \multirow{5}{*}{1.23} & & - & Control \\
\hline & & & & & & + & $\mathrm{nCeO} 2$ \\
\hline & & & & & 92 & + & $\mathrm{G}-\mathrm{CeO}_{2}$ \\
\hline & & & & & & - & $\mathrm{L}-\mathrm{CeO}_{2}$ \\
\hline & & & & & & - & $\mathrm{P}-\mathrm{CeO}_{2}$ \\
\hline & & & & & & + & Control \\
\hline 2,4-bis(1,1- & & & & & & + & $\mathrm{nCeO} 2$ \\
\hline dimethylethyl)- & $\mathrm{C}_{16} \mathrm{H}_{26} \mathrm{O}$ & 80 & 32.251 & 1.53 & 93 & + & G-CeO ${ }_{2}$ \\
\hline & & & & & & + & $\mathrm{L}-\mathrm{CeO}_{2}$ \\
\hline & & & & & & + & $\mathrm{P}-\mathrm{CeO}_{2}$ \\
\hline & & & & & & + & Control \\
\hline 3,7,11-trimethyl- & & & & & & + & $\mathrm{nCeO} 2$ \\
\hline $2,6,10-$ & $\mathrm{C}_{15} \mathrm{H}_{26} \mathrm{O}$ & 81 & 32.650 & 1.44 & 86 & + & $\mathrm{G}-\mathrm{CeO}_{2}$ \\
\hline dodecatrien-1-ol & & & & & & + & $\mathrm{L}-\mathrm{CeO}_{2}$ \\
\hline & & & & & & + & $\mathrm{P}-\mathrm{CeO}_{2}$ \\
\hline & & & & & & + & Control \\
\hline & & & & & & + & $\mathrm{nCeO} 2$ \\
\hline $\begin{array}{l}\text { L-metny-1- } \\
\text { pentadecene }\end{array}$ & $\mathrm{C}_{16} \mathrm{H}_{32}$ & 82 & 33.044 & 1.11 & 81 & + & G-CeO ${ }_{2}$ \\
\hline & & & & & & + & $\mathrm{L}-\mathrm{CeO}_{2}$ \\
\hline & & & & & & + & $\mathrm{P}-\mathrm{CeO}_{2}$ \\
\hline & & & & & & + & Control \\
\hline Diethyl-1,2- & & & & & & + & $\mathrm{nCeO} 2$ \\
\hline benzenedicarboxy & $\mathrm{C}_{12} \mathrm{H}_{14} \mathrm{O}_{4}$ & 83 & 33.767 & 2.46 & 89 & + & $\mathrm{G}-\mathrm{CeO}_{2}$ \\
\hline & & & & & & + & $\mathrm{L}-\mathrm{CeO}_{2}$ \\
\hline & & & & & & + & $\mathrm{P}-\mathrm{CeO}_{2}$ \\
\hline & & & & & & - & Control \\
\hline 4-(2,6,6-trimethyl- & & & & & & + & $\mathrm{nCeO} 2$ \\
\hline 1-cyclohexen-1- & $\mathrm{C}_{13} \mathrm{H}_{20} \mathrm{O}$ & 84 & 34.555 & 1.77 & 82 & - & G-CeO ${ }_{2}$ \\
\hline yl)-3-buten-2-one & & & & & & - & $\mathrm{L}-\mathrm{CeO}_{2}$ \\
\hline & & & & & & + & $\mathrm{P}-\mathrm{CeO}_{2}$ \\
\hline & & & & & & + & Control \\
\hline & & & & & & + & $\mathrm{nCeO} 2$ \\
\hline Tetradecanal & $\mathrm{C}_{14} \mathrm{H}_{28} \mathrm{O}$ & 85 & 34.647 & 1.26 & 95 & + & G-CeO ${ }_{2}$ \\
\hline & & & & & & + & $\mathrm{L}-\mathrm{CeO}_{2}$ \\
\hline & & & & & & + & $\mathrm{P}-\mathrm{CeO}_{2}$ \\
\hline & & & & & & + & Control \\
\hline & & & & & & + & $\mathrm{nCeO} 2$ \\
\hline $\begin{array}{l}\text { Isopropyi } \\
\text { dodecanoate }\end{array}$ & $\mathrm{C}_{15} \mathrm{H}_{30} \mathrm{O}_{2}$ & 86 & 35.045 & 1.14 & 91 & + & G-CeO ${ }_{2}$ \\
\hline & & & & & & + & $\mathrm{L}-\mathrm{CeO}_{2}$ \\
\hline & & & & & & + & $\mathrm{P}-\mathrm{CeO}_{2}$ \\
\hline Methyl-(3-oxo-2- & & & & & & + & Control \\
\hline pentyl-, & $\mathrm{C}_{13} \mathrm{H}_{22} \mathrm{O}_{3}$ & 87 & 35.357 & 1.89 & 91 & + & $\mathrm{nCeO} 2$ \\
\hline cyclopentyl)acetat & & & & & & + & $\mathrm{G}-\mathrm{CeO}_{2}$ \\
\hline
\end{tabular}




\begin{tabular}{|c|c|c|c|c|c|c|c|}
\hline Compounds & Formula & $\begin{array}{c}\text { Order } \\
\text { number }\end{array}$ & RT1 & RT2 & $\begin{array}{c}\text { Similarity } \\
(\%)\end{array}$ & Presence & Treatments \\
\hline \multirow[t]{2}{*}{$\mathrm{e}$} & & & & & & + & $\mathrm{L}-\mathrm{CeO}_{2}$ \\
\hline & & & & & & + & $\mathrm{P}-\mathrm{CeO}_{2}$ \\
\hline \multirow{5}{*}{ 2-tetradecanol } & \multirow{5}{*}{$\mathrm{C}_{14} \mathrm{H}_{30} \mathrm{O}$} & \multirow{5}{*}{88} & \multirow{5}{*}{36.043} & \multirow{5}{*}{1.02} & \multirow{5}{*}{84} & + & Control \\
\hline & & & & & & - & $\mathrm{nCeO} 2$ \\
\hline & & & & & & - & $\mathrm{G}-\mathrm{CeO}_{2}$ \\
\hline & & & & & & - & $\mathrm{L}-\mathrm{CeO}_{2}$ \\
\hline & & & & & & - & $\mathrm{P}-\mathrm{CeO}_{2}$ \\
\hline \multirow{5}{*}{$\begin{array}{c}\text { 1-(4- } \\
\text { isopropylphenyl)- } \\
\text { 2-methylpropyl } \\
\text { acetate }\end{array}$} & \multirow{5}{*}{$\mathrm{C}_{15} \mathrm{H}_{22} \mathrm{O}_{2}$} & \multirow{5}{*}{89} & \multirow{5}{*}{36.153} & \multirow{5}{*}{1.62} & \multirow{5}{*}{86} & + & Control \\
\hline & & & & & & + & $\mathrm{nCeO} 2$ \\
\hline & & & & & & + & $\mathrm{G}-\mathrm{CeO}_{2}$ \\
\hline & & & & & & + & $\mathrm{L}-\mathrm{CeO}_{2}$ \\
\hline & & & & & & + & $\mathrm{P}-\mathrm{CeO}_{2}$ \\
\hline \multirow{5}{*}{$n$-hexyl salicylate } & \multirow{5}{*}{$\mathrm{C}_{13} \mathrm{H}_{18} \mathrm{O}_{3}$} & \multirow{5}{*}{90} & \multirow{5}{*}{36.154} & & & + & Control \\
\hline & & & & & & + & $\mathrm{nCeO} 2$ \\
\hline & & & & 1.71 & 89 & + & $\mathrm{G}-\mathrm{CeO}_{2}$ \\
\hline & & & & & & + & $\mathrm{L}-\mathrm{CeO}_{2}$ \\
\hline & & & & & & + & $\mathrm{P}-\mathrm{CeO}_{2}$ \\
\hline & & & & & & + & Control \\
\hline & & & & & & + & $\mathrm{nCeO} 2$ \\
\hline 1-tetradecanol & $\mathrm{C}_{14} \mathrm{H}_{30} \mathrm{O}$ & 91 & 36.447 & 1.26 & 90 & + & $\mathrm{G}-\mathrm{CeO}_{2}$ \\
\hline & & & & & & + & $\mathrm{L}-\mathrm{CeO}_{2}$ \\
\hline & & & & & & + & $\mathrm{P}-\mathrm{CeO}_{2}$ \\
\hline & & & & & & + & Control \\
\hline & & & & & & + & $\mathrm{nCeO} 2$ \\
\hline 13-tetradecenal & $\mathrm{C}_{14} \mathrm{H}_{26} \mathrm{O}$ & 92 & 36.549 & 1.38 & 87 & + & $\mathrm{G}-\mathrm{CeO}_{2}$ \\
\hline & & & & & & + & $\mathrm{L}-\mathrm{CeO}_{2}$ \\
\hline & & & & & & + & $\mathrm{P}-\mathrm{CeO}_{2}$ \\
\hline & & & & & & + & Control \\
\hline & & & & & & + & $\mathrm{nCeO} 2$ \\
\hline Pentadecanal & $\mathrm{C}_{15} \mathrm{H}_{30} \mathrm{O}$ & 93 & 36.947 & 1.29 & 95 & + & $\mathrm{G}-\mathrm{CeO}_{2}$ \\
\hline & & & & & & + & $\mathrm{L}-\mathrm{CeO}_{2}$ \\
\hline & & & & & & + & $\mathrm{P}-\mathrm{CeO}_{2}$ \\
\hline 2,6-di-t-butyl-4- & & & & & & + & Control \\
\hline hydroxymethylene & & & & & & + & $\mathrm{nCeO} 2$ \\
\hline$-2,3,5,6-$ & $\mathrm{C}_{15} \mathrm{H}_{22} \mathrm{O}_{2}$ & 94 & 37.860 & 2.04 & 85 & + & $\mathrm{G}-\mathrm{CeO}_{2}$ \\
\hline detetrahydrocyclo & & & & & & + & $\mathrm{L}-\mathrm{CeO}_{2}$ \\
\hline hexanone & & & & & & + & $\mathrm{P}-\mathrm{CeO}_{2}$ \\
\hline & & & & & & - & Control \\
\hline 2,3,6-trimethyl- & & & & & & + & $\mathrm{nCeO} 2$ \\
\hline 1,4 & $\mathrm{C}_{13} \mathrm{H}_{12} \mathrm{O}_{2}$ & 95 & 37.871 & 2.67 & 80 & + & G-CeO ${ }_{2}$ \\
\hline naphthalenedione & & & & & & + & $\mathrm{L}-\mathrm{CeO}_{2}$ \\
\hline & & & & & & + & $\mathrm{P}-\mathrm{CeO}_{2}$ \\
\hline & & & & & & + & Control \\
\hline & & & & & & + & $\mathrm{nCeO} 2$ \\
\hline Pentyl benzoate & $\mathrm{C}_{12} \mathrm{H}_{16} \mathrm{O}_{2}$ & 96 & 38.553 & 1.65 & 81 & + & G-CeO ${ }_{2}$ \\
\hline & & & & & & + & $\mathrm{L}-\mathrm{CeO}_{2}$ \\
\hline & & & & & & + & $\mathrm{P}-\mathrm{CeO}_{2}$ \\
\hline & & & & & & + & Control \\
\hline & & & & & & + & $\mathrm{nCeO} 2$ \\
\hline $\begin{array}{l}\text { 2-metny1-2- } \\
\text { phenvl-tridecane }\end{array}$ & $\mathrm{C}_{20} \mathrm{H}_{34}$ & 97 & 38.661 & 2.10 & 84 & + & G-CeO ${ }_{2}$ \\
\hline & & & & & & + & $\mathrm{L}-\mathrm{CeO}_{2}$ \\
\hline & & & & & & + & $\mathrm{P}-\mathrm{CeO}_{2}$ \\
\hline & & & & & & + & Control \\
\hline Hexadecanal & $\mathrm{C}_{16} \mathrm{H}_{32} \mathrm{O}$ & 98 & 39.048 & 1.32 & 94 & + & $\mathrm{nCeO} 2$ \\
\hline & & & & & & + & $\mathrm{G}-\mathrm{CeO}_{2}$ \\
\hline
\end{tabular}




\begin{tabular}{|c|c|c|c|c|c|c|c|}
\hline Compounds & Formula & $\begin{array}{c}\text { Order } \\
\text { number }\end{array}$ & RT1 & RT2 & $\begin{array}{c}\text { Similarity } \\
(\%)\end{array}$ & Presence & Treatments \\
\hline & & & & & & + & $\mathrm{L}-\mathrm{CeO}_{2}$ \\
\hline & & & & & & + & $\mathrm{P}-\mathrm{CeO}_{2}$ \\
\hline \multirow{5}{*}{$\begin{array}{l}\mathrm{N}, \mathrm{N} \text {-diethyl-1- } \\
\text { dodecaneamine }\end{array}$} & \multirow{5}{*}{$\mathrm{C}_{14} \mathrm{H}_{31} \mathrm{~N}$} & \multirow{5}{*}{99} & \multirow{5}{*}{39.450} & \multirow{5}{*}{1.44} & \multirow{5}{*}{89} & + & Control \\
\hline & & & & & & + & $\mathrm{nCeO} 2$ \\
\hline & & & & & & + & G-CeO ${ }_{2}$ \\
\hline & & & & & & + & $\mathrm{L}-\mathrm{CeO}_{2}$ \\
\hline & & & & & & + & $\mathrm{P}-\mathrm{CeO}_{2}$ \\
\hline \multirow{5}{*}{$\begin{array}{c}\text { bis(2- } \\
\text { methylpropyl)- } \\
1,2- \\
\text { benzenedicarboxy } \\
\text { late } \\
\end{array}$} & \multirow{5}{*}{$\mathrm{C}_{16} \mathrm{H}_{22} \mathrm{O}_{4}$} & \multirow{5}{*}{100} & \multirow{5}{*}{39.762} & \multirow{5}{*}{2.16} & \multirow{5}{*}{96} & + & Control \\
\hline & & & & & & + & $\mathrm{nCeO} 2$ \\
\hline & & & & & & + & $\mathrm{G}-\mathrm{CeO}_{2}$ \\
\hline & & & & & & + & $\mathrm{L}-\mathrm{CeO}_{2}$ \\
\hline & & & & & & + & $\mathrm{P}-\mathrm{CeO}_{2}$ \\
\hline \multirow{5}{*}{ Heptyl benzoate } & \multirow{5}{*}{$\mathrm{C}_{14} \mathrm{H}_{20} \mathrm{O}_{2}$} & \multirow{5}{*}{101} & \multirow{5}{*}{39.955} & \multirow{5}{*}{1.77} & \multirow{5}{*}{82} & + & Control \\
\hline & & & & & & + & $\mathrm{nCeO} 2$ \\
\hline & & & & & & + & G-CeO ${ }_{2}$ \\
\hline & & & & & & + & $\mathrm{L}-\mathrm{CeO}_{2}$ \\
\hline & & & & & & + & $\mathrm{P}-\mathrm{CeO}_{2}$ \\
\hline \multirow{5}{*}{ Tridecyl benzoate } & & & & & & + & Control \\
\hline & & & & & & + & $\mathrm{nCeO} 2$ \\
\hline & $\mathrm{C}_{20} \mathrm{H}_{32} \mathrm{O}_{2}$ & 102 & 40.455 & 1.77 & 87 & + & G-CeO ${ }_{2}$ \\
\hline & & & & & & + & $\mathrm{L}-\mathrm{CeO}_{2}$ \\
\hline & & & & & & + & $\mathrm{P}-\mathrm{CeO}_{2}$ \\
\hline & & & & & & + & Control \\
\hline & & & & & & + & $\mathrm{nCeO} 2$ \\
\hline$n$-hexadecanoic & $\mathrm{C}_{16} \mathrm{H}_{32} \mathrm{O}_{2}$ & 103 & 41.849 & 1.41 & 92 & + & G-CeO ${ }_{2}$ \\
\hline & & & & & & + & $\mathrm{L}-\mathrm{CeO}_{2}$ \\
\hline & & & & & & + & $\mathrm{P}-\mathrm{CeO}_{2}$ \\
\hline & & & & & & + & Control \\
\hline & & & & & & + & $\mathrm{nCeO} 2$ \\
\hline cis,cis,cis- $7,10,13-$ & $\mathrm{C}_{16} \mathrm{H}_{26} \mathrm{O}$ & 104 & 42.454 & 1.65 & 91 & + & G-CeO ${ }_{2}$ \\
\hline & & & & & & + & $\mathrm{L}-\mathrm{CeO}_{2}$ \\
\hline & & & & & & + & $\mathrm{P}-\mathrm{CeO}_{2}$ \\
\hline & & & & & & - & Control \\
\hline & & & & & & - & $\mathrm{nCeO} 2$ \\
\hline $\begin{array}{l}\text { Ethyl } \\
\text { hentadecanoate }\end{array}$ & $\mathrm{C}_{19} \mathrm{H}_{38} \mathrm{O}_{2}$ & 105 & 42.547 & 1.29 & 83 & + & G-CeO ${ }_{2}$ \\
\hline & & & & & & + & $\mathrm{L}-\mathrm{CeO}_{2}$ \\
\hline & & & & & & + & $\mathrm{P}-\mathrm{CeO}_{2}$ \\
\hline & & & & & & + & Control \\
\hline & & & & & & + & $\mathrm{nCeO} 2$ \\
\hline (Z)-9-Octadecenal & $\mathrm{C}_{18} \mathrm{H}_{34} \mathrm{O}$ & 106 & 42.550 & 1.47 & 92 & + & G-CeO ${ }_{2}$ \\
\hline & & & & & & + & $\mathrm{L}-\mathrm{CeO}_{2}$ \\
\hline & & & & & & + & $\mathrm{P}-\mathrm{CeO}_{2}$ \\
\hline & & & & & & + & Control \\
\hline & & & & & & + & $\mathrm{nCeO} 2$ \\
\hline Heptadecanal & $\mathrm{C}_{17} \mathrm{H}_{34} \mathrm{O}$ & 107 & 43.049 & 1.38 & 94 & + & G-CeO ${ }_{2}$ \\
\hline & & & & & & - & $\mathrm{L}-\mathrm{CeO}_{2}$ \\
\hline & & & & & & + & $\mathrm{P}-\mathrm{CeO}_{2}$ \\
\hline & & & & & & - & Control \\
\hline & & & & & & - & $\mathrm{nCeO} 2$ \\
\hline $\begin{array}{c}2- \\
\text { methyltetracosane }\end{array}$ & $\mathrm{C}_{25} \mathrm{H}_{52}$ & 108 & 56.669 & 2.58 & 86 & - & G-CeO ${ }_{2}$ \\
\hline & & & & & & + & $\mathrm{L}-\mathrm{CeO}_{2}$ \\
\hline & & & & & & + & $\mathrm{P}-\mathrm{CeO}_{2}$ \\
\hline
\end{tabular}


Supplementary Table S2. Compounds tentatively identified by software of GCxGC-MS method in methylene chloride extracts of pea shoot; + means presence and - means absence of compounds in the sample

\begin{tabular}{|c|c|c|c|c|c|c|c|}
\hline Compounds & Formula & $\begin{array}{c}\text { Order } \\
\text { number }\end{array}$ & RT1 & RT2 & $\underset{(\%)}{\text { Similarity }}$ & Presence & Treatments \\
\hline \multirow{5}{*}{$\begin{array}{l}\text { 1,2-dimethyl- } \\
\text { benzene }\end{array}$} & \multirow{5}{*}{$\mathrm{C}_{8} \mathrm{H}_{10}$} & \multirow{5}{*}{1} & \multirow{5}{*}{12.769} & \multirow{5}{*}{2.64} & \multirow{5}{*}{92} & - & Control \\
\hline & & & & & & - & $\mathrm{nCeO} 2$ \\
\hline & & & & & & - & G-CeO ${ }_{2}$ \\
\hline & & & & & & + & $\mathrm{L}-\mathrm{CeO}_{2}$ \\
\hline & & & & & & + & $\mathrm{P}-\mathrm{CeO}_{2}$ \\
\hline \multirow{5}{*}{$\begin{array}{l}\text { 2,3,4-trimethyl- } \\
\text { hexane }\end{array}$} & \multirow{5}{*}{$\mathrm{C}_{9} \mathrm{H}_{20}$} & \multirow{5}{*}{2} & \multirow{5}{*}{12.948} & \multirow{5}{*}{1.35} & \multirow{5}{*}{80} & - & Control \\
\hline & & & & & & - & $\mathrm{nCeO} 2$ \\
\hline & & & & & & - & G-CeO ${ }_{2}$ \\
\hline & & & & & & + & $\mathrm{L}-\mathrm{CeO}_{2}$ \\
\hline & & & & & & - & $\mathrm{P}-\mathrm{CeO}_{2}$ \\
\hline \multirow{5}{*}{$\begin{array}{c}(1,2- \\
\text { dimethylbutyl)- } \\
\text { cyclohexane }\end{array}$} & \multirow{5}{*}{$\mathrm{C}_{12} \mathrm{H}_{24}$} & \multirow{5}{*}{3} & \multirow{5}{*}{14.054} & \multirow{5}{*}{1.77} & \multirow{5}{*}{84} & - & Control \\
\hline & & & & & & - & $\mathrm{nCeO} 2$ \\
\hline & & & & & & - & G-CeO ${ }_{2}$ \\
\hline & & & & & & - & $\mathrm{L}-\mathrm{CeO}_{2}$ \\
\hline & & & & & & + & $\mathrm{P}-\mathrm{CeO}_{2}$ \\
\hline \multirow{5}{*}{$\begin{array}{c}\text { Cyclopentane-1,2- } \\
\text { diol }\end{array}$} & \multirow{5}{*}{$\mathrm{C}_{5} \mathrm{H}_{10} \mathrm{O}_{2}$} & & & & & - & Control \\
\hline & & & & & & - & $\mathrm{nCeO} 2$ \\
\hline & & 4 & 14.858 & 1.95 & 85 & + & $\mathrm{G}-\mathrm{CeO}_{2}$ \\
\hline & & & & & & + & $\mathrm{L}-\mathrm{CeO}_{2}$ \\
\hline & & & & & & + & $\mathrm{P}-\mathrm{CeO}_{2}$ \\
\hline & & & & & & - & Control \\
\hline & & & & & & - & $\mathrm{nCeO} 2$ \\
\hline (E)-2-heptenal & $\mathrm{C}_{7} \mathrm{H}_{12} \mathrm{O}$ & 5 & 15.651 & 1.56 & 86 & - & $\mathrm{G}-\mathrm{CeO}_{2}$ \\
\hline & & & & & & + & $\mathrm{L}-\mathrm{CeO}_{2}$ \\
\hline & & & & & & + & $\mathrm{P}-\mathrm{CeO}_{2}$ \\
\hline & & & & & & - & Control \\
\hline & & & & & & - & $\mathrm{nCeO} 2$ \\
\hline Benzaldehyde & $\mathrm{C}_{7} \mathrm{H}_{6} \mathrm{O}$ & 6 & 15.664 & 2.31 & 82 & + & G-CeO ${ }_{2}$ \\
\hline & & & & & & + & $\mathrm{L}-\mathrm{CeO}_{2}$ \\
\hline & & & & & & + & $\mathrm{P}-\mathrm{CeO}_{2}$ \\
\hline & & & & & & - & Control \\
\hline & & & & & & - & $\mathrm{nCeO} 2$ \\
\hline 3,1-dimethyl-1- & $\mathrm{C}_{10} \mathrm{H}_{20}$ & 7 & 15.961 & 2.16 & 88 & + & $\mathrm{G}-\mathrm{CeO}_{2}$ \\
\hline & & & & & & + & $\mathrm{L}-\mathrm{CeO}_{2}$ \\
\hline & & & & & & + & $\mathrm{P}-\mathrm{CeO}_{2}$ \\
\hline & & & & & & - & Control \\
\hline & & & & & & - & $\mathrm{nCeO} 2$ \\
\hline $\begin{array}{l}(E, E)-2,4- \\
\text { hentadienal }\end{array}$ & $\mathrm{C}_{7} \mathrm{H}_{10} \mathrm{O}$ & 8 & 17.054 & 1.74 & 88 & - & G-CeO ${ }_{2}$ \\
\hline & & & & & & + & $\mathrm{L}-\mathrm{CeO}_{2}$ \\
\hline & & & & & & + & $\mathrm{P}-\mathrm{CeO}_{2}$ \\
\hline & & & & & & - & Control \\
\hline & & & & & & - & $\mathrm{nCeO} 2$ \\
\hline Octanal & $\mathrm{C}_{8} \mathrm{H}_{16} \mathrm{O}$ & 9 & 17.346 & 1.23 & 82 & + & $\mathrm{G}-\mathrm{CeO}_{2}$ \\
\hline & & & & & & + & $\mathrm{L}-\mathrm{CeO}_{2}$ \\
\hline & & & & & & + & $\mathrm{P}-\mathrm{CeO}_{2}$ \\
\hline & & & & & & - & Control \\
\hline & & & & & & - & $\mathrm{nCeO} 2$ \\
\hline 2-ethyl-1-hexanol & $\mathrm{C}_{8} \mathrm{H}_{18} \mathrm{O}$ & 10 & 18.544 & 1.11 & 82 & - & $\mathrm{G}-\mathrm{CeO}_{2}$ \\
\hline & & & & & & + & $\mathrm{L}-\mathrm{CeO}_{2}$ \\
\hline & & & & & & + & $\mathrm{P}-\mathrm{CeO}_{2}$ \\
\hline
\end{tabular}




\begin{tabular}{|c|c|c|c|c|c|c|c|}
\hline Compounds & Formula & $\begin{array}{c}\text { Order } \\
\text { number }\end{array}$ & RT1 & RT2 & $\begin{array}{c}\text { Similarity } \\
(\%)\end{array}$ & Presence & Treatments \\
\hline \multirow{5}{*}{$\begin{array}{l}\text { 3,7-dimethyl-2,6- } \\
\text { octadien-1-ol }\end{array}$} & \multirow{5}{*}{$\mathrm{C}_{10} \mathrm{H}_{18} \mathrm{O}$} & \multirow{5}{*}{11} & \multirow{5}{*}{19.147} & \multirow{5}{*}{1.32} & \multirow{5}{*}{81} & - & Control \\
\hline & & & & & & - & $\mathrm{nCeO} 2$ \\
\hline & & & & & & + & $\mathrm{G}-\mathrm{CeO}_{2}$ \\
\hline & & & & & & + & $\mathrm{L}-\mathrm{CeO}_{2}$ \\
\hline & & & & & & + & $\mathrm{P}-\mathrm{CeO}_{2}$ \\
\hline \multirow{5}{*}{$\begin{array}{c}\text { 2-methyl- } \\
\text { benzaldehyde }\end{array}$} & \multirow{5}{*}{$\mathrm{C}_{8} \mathrm{H}_{8} \mathrm{O}$} & \multirow{5}{*}{12} & \multirow{5}{*}{19.460} & \multirow{5}{*}{2.10} & \multirow{5}{*}{88} & - & Control \\
\hline & & & & & & - & $\mathrm{nCeO} 2$ \\
\hline & & & & & & - & $\mathrm{G}-\mathrm{CeO}_{2}$ \\
\hline & & & & & & + & $\mathrm{L}-\mathrm{CeO}_{2}$ \\
\hline & & & & & & + & $\mathrm{P}-\mathrm{CeO}_{2}$ \\
\hline \multirow{5}{*}{ 4-methyl-phenol } & \multirow{5}{*}{$\mathrm{C}_{7} \mathrm{H}_{8} \mathrm{O}$} & \multirow{5}{*}{13} & \multirow{5}{*}{19.856} & \multirow{5}{*}{1.83} & & - & Control \\
\hline & & & & & & - & $\mathrm{nCeO} 2$ \\
\hline & & & & & 90 & + & $\mathrm{G}-\mathrm{CeO}_{2}$ \\
\hline & & & & & & + & $\mathrm{L}-\mathrm{CeO}_{2}$ \\
\hline & & & & & & + & $\mathrm{P}-\mathrm{CeO}_{2}$ \\
\hline & & & & & & - & Control \\
\hline & & & & & & - & $\mathrm{nCeO} 2$ \\
\hline $\begin{array}{l}\text { 4-methyl- } \\
\text { henzaldehyde }\end{array}$ & $\mathrm{C}_{8} \mathrm{H}_{8} \mathrm{O}$ & 14 & 19.861 & 2.13 & 96 & + & G-CeO ${ }_{2}$ \\
\hline & & & & & & + & $\mathrm{L}-\mathrm{CeO}_{2}$ \\
\hline & & & & & & + & $\mathrm{P}-\mathrm{CeO}_{2}$ \\
\hline & & & & & & - & Control \\
\hline & & & & & & - & $\mathrm{nCeO} 2$ \\
\hline Cyclohexanol & $\mathrm{C}_{6} \mathrm{H}_{12} \mathrm{O}$ & 15 & 20.144 & 1.08 & 82 & + & G-CeO ${ }_{2}$ \\
\hline & & & & & & + & $\mathrm{L}-\mathrm{CeO}_{2}$ \\
\hline & & & & & & + & $\mathrm{P}-\mathrm{CeO}_{2}$ \\
\hline & & & & & & - & Control \\
\hline & & & & & & - & $\mathrm{nCeO} 2$ \\
\hline $\begin{array}{l}4,5 \text {-aimetnyl-1- } \\
\text { hexene }\end{array}$ & $\mathrm{C}_{8} \mathrm{H}_{16}$ & 16 & 20.246 & 1.23 & 82 & + & G-CeO ${ }_{2}$ \\
\hline & & & & & & + & $\mathrm{L}-\mathrm{CeO}_{2}$ \\
\hline & & & & & & + & $\mathrm{P}-\mathrm{CeO}_{2}$ \\
\hline & & & & & & - & Control \\
\hline 1-methyl-4-(1- & & & & & & - & $\mathrm{nCeO} 2$ \\
\hline methylethyl)- & $\mathrm{C}_{10} \mathrm{H}_{14}$ & 17 & 20.446 & 1.26 & 81 & + & $\mathrm{G}-\mathrm{CeO}_{2}$ \\
\hline benzene & & & & & & + & $\mathrm{L}-\mathrm{CeO}_{2}$ \\
\hline & & & & & & + & $\mathrm{P}-\mathrm{CeO}_{2}$ \\
\hline & & & & & & - & Control \\
\hline & & & & & & - & $\mathrm{nCeO} 2$ \\
\hline Nonanal & $\mathrm{C}_{9} \mathrm{H}_{18} \mathrm{O}$ & 18 & 20.845 & 1.17 & 95 & + & G-CeO ${ }_{2}$ \\
\hline & & & & & & + & $\mathrm{L}-\mathrm{CeO}_{2}$ \\
\hline & & & & & & + & $\mathrm{P}-\mathrm{CeO}_{2}$ \\
\hline & & & & & & - & Control \\
\hline $1,2,3,4-$ & & & & & & - & $\mathrm{nCeO} 2$ \\
\hline tetramethyl- & $\mathrm{C}_{10} \mathrm{H}_{14}$ & 19 & 21.647 & 1.32 & 92 & + & $\mathrm{G}-\mathrm{CeO}_{2}$ \\
\hline benzene & & & & & & + & $\mathrm{L}-\mathrm{CeO}_{2}$ \\
\hline & & & & & & - & $\mathrm{P}-\mathrm{CeO}_{2}$ \\
\hline & & & & & & - & Control \\
\hline 1-methyl-2- & & & & & & - & $\mathrm{nCeO} 2$ \\
\hline phenylcyclopropa & $\mathrm{C}_{10} \mathrm{H}_{12}$ & 20 & 22.151 & 1.53 & 80 & + & G-CeO ${ }_{2}$ \\
\hline & & & & & & + & $\mathrm{L}-\mathrm{CeO}_{2}$ \\
\hline & & & & & & - & $\mathrm{P}-\mathrm{CeO}_{2}$ \\
\hline & & & & & & - & Control \\
\hline & & & & & & - & $\mathrm{nCeO} 2$ \\
\hline $\begin{array}{c}\text { 1-ethyl-2,3- } \\
\text { dimethyl-henzene }\end{array}$ & $\mathrm{C}_{10} \mathrm{H}_{14}$ & 21 & 22.649 & 1.41 & 89 & + & $\mathrm{G}-\mathrm{CeO}_{2}$ \\
\hline & & & & & & + & $\mathrm{L}-\mathrm{CeO}_{2}$ \\
\hline & & & & & & - & $\mathrm{P}-\mathrm{CeO}_{2}$ \\
\hline
\end{tabular}




\begin{tabular}{|c|c|c|c|c|c|c|c|}
\hline Compounds & Formula & $\begin{array}{c}\text { Order } \\
\text { number }\end{array}$ & RT1 & RT2 & $\begin{array}{c}\text { Similarity } \\
(\%)\end{array}$ & Presence & Treatments \\
\hline \multirow{5}{*}{ Octanoic acid } & \multirow{5}{*}{$\mathrm{C}_{8} \mathrm{H}_{16} \mathrm{O}_{2}$} & \multirow{5}{*}{22} & \multirow{5}{*}{22.945} & \multirow{5}{*}{1.20} & \multirow{5}{*}{89} & + & Control \\
\hline & & & & & & - & $\mathrm{nCeO} 2$ \\
\hline & & & & & & + & G-CeO ${ }_{2}$ \\
\hline & & & & & & + & $\mathrm{L}-\mathrm{CeO}_{2}$ \\
\hline & & & & & & + & $\mathrm{P}-\mathrm{CeO}_{2}$ \\
\hline \multirow{5}{*}{ Naphthalene } & \multirow{5}{*}{$\mathrm{C}_{10} \mathrm{H}_{8}$} & \multirow{5}{*}{23} & \multirow{5}{*}{23.460} & \multirow{5}{*}{2.10} & \multirow{5}{*}{94} & - & Control \\
\hline & & & & & & - & $\mathrm{nCeO} 2$ \\
\hline & & & & & & + & $\mathrm{G}-\mathrm{CeO}_{2}$ \\
\hline & & & & & & + & $\mathrm{L}-\mathrm{CeO}_{2}$ \\
\hline & & & & & & + & $\mathrm{P}-\mathrm{CeO}_{2}$ \\
\hline \multirow{5}{*}{ Decanal } & \multirow{5}{*}{$\mathrm{C}_{10} \mathrm{H}_{20} \mathrm{O}$} & \multirow{5}{*}{24} & \multirow{5}{*}{23.945} & \multirow{5}{*}{1.17} & & - & Control \\
\hline & & & & & & - & $\mathrm{nCeO} 2$ \\
\hline & & & & & 94 & + & $\mathrm{G}-\mathrm{CeO}_{2}$ \\
\hline & & & & & & + & $\mathrm{L}-\mathrm{CeO}_{2}$ \\
\hline & & & & & & + & $\mathrm{P}-\mathrm{CeO}_{2}$ \\
\hline & & & & & & - & Control \\
\hline $1,2,3,4,5-$ & & & & & & - & $\mathrm{nCeO} 2$ \\
\hline pentamethyl- & $\mathrm{C}_{11} \mathrm{H}_{16}$ & 25 & 24.047 & 1.32 & 83 & + & G-CeO ${ }_{2}$ \\
\hline benzene & & & & & & + & $\mathrm{L}-\mathrm{CeO}_{2}$ \\
\hline & & & & & & - & $\mathrm{P}-\mathrm{CeO}_{2}$ \\
\hline & & & & & & - & Control \\
\hline & & & & & & - & $\mathrm{nCeO} 2$ \\
\hline $\begin{array}{l}\text { 2,4-dimethyl- } \\
\text { henzaldehyde }\end{array}$ & $\mathrm{C}_{9} \mathrm{H}_{10} \mathrm{O}$ & 26 & 24.160 & 2.13 & 96 & + & G-CeO ${ }_{2}$ \\
\hline & & & & & & + & $\mathrm{L}-\mathrm{CeO}_{2}$ \\
\hline & & & & & & + & $\mathrm{P}-\mathrm{CeO}_{2}$ \\
\hline & & & & & & - & Control \\
\hline & & & & & & - & $\mathrm{nCeO} 2$ \\
\hline Benzothiazole & $\mathrm{C}_{7} \mathrm{H}_{5} \mathrm{NS}$ & 27 & 24.374 & 2.88 & 87 & + & G-CeO ${ }_{2}$ \\
\hline & & & & & & + & $\mathrm{L}-\mathrm{CeO}_{2}$ \\
\hline & & & & & & + & $\mathrm{P}-\mathrm{CeO}_{2}$ \\
\hline & & & & & & - & Control \\
\hline 2,6,6-trimethyl-1- & & & & & & - & $\mathrm{nCeO} 2$ \\
\hline cyclohexene-1- & $\mathrm{C}_{10} \mathrm{H}_{16} \mathrm{O}$ & 28 & 24.453 & 1.65 & 87 & - & G-CeO ${ }_{2}$ \\
\hline & & & & & & + & $\mathrm{L}-\mathrm{CeO}_{2}$ \\
\hline & & & & & & + & $\mathrm{P}-\mathrm{CeO}_{2}$ \\
\hline & & & & & & - & Control \\
\hline & & & & & & - & $\mathrm{nCeO} 2$ \\
\hline (E)-2-decenal & $\mathrm{C}_{10} \mathrm{H}_{18} \mathrm{O}$ & 29 & 25.547 & 1.32 & 90 & + & $\mathrm{G}-\mathrm{CeO}_{2}$ \\
\hline & & & & & & + & $\mathrm{L}-\mathrm{CeO}_{2}$ \\
\hline & & & & & & + & $\mathrm{P}-\mathrm{CeO}_{2}$ \\
\hline & & & & & & + & Control \\
\hline & & & & & & - & $\mathrm{nCeO} 2$ \\
\hline Nonanoic acid & $\mathrm{C}_{9} \mathrm{H}_{18} \mathrm{O}_{2}$ & 30 & 25.746 & 1.23 & 85 & + & $\mathrm{G}-\mathrm{CeO}_{2}$ \\
\hline & & & & & & + & $\mathrm{L}-\mathrm{CeO}_{2}$ \\
\hline & & & & & & + & $\mathrm{P}-\mathrm{CeO}_{2}$ \\
\hline & & & & & & - & Control \\
\hline & & & & & & - & $\mathrm{nCeO} 2$ \\
\hline 2-hydroxy-1so- & $\mathrm{C}_{10} \mathrm{H}_{12} \mathrm{O}_{2}$ & 31 & 26.061 & 2.13 & 92 & + & G-CeO ${ }_{2}$ \\
\hline & & & & & & + & $\mathrm{L}-\mathrm{CeO}_{2}$ \\
\hline & & & & & & + & $\mathrm{P}-\mathrm{CeO}_{2}$ \\
\hline & & & & & & - & Control \\
\hline & & & & & & - & $\mathrm{nCeO} 2$ \\
\hline $\begin{array}{c}\text { Methyl-1,2- } \\
\text { benzenecarboxylate }\end{array}$ & $\mathrm{C}_{9} \mathrm{H}_{7} \mathrm{O}_{4}$ & 32 & 26.380 & 3.30 & 93 & + & $\mathrm{G}-\mathrm{CeO}_{2}$ \\
\hline & & & & & & + & $\mathrm{L}-\mathrm{CeO}_{2}$ \\
\hline & & & & & & + & $\mathrm{P}-\mathrm{CeO}_{2}$ \\
\hline
\end{tabular}




\begin{tabular}{|c|c|c|c|c|c|c|c|}
\hline Compounds & Formula & $\begin{array}{c}\text { Order } \\
\text { number }\end{array}$ & RT1 & RT2 & $\begin{array}{c}\text { Similarity } \\
(\%)\end{array}$ & Presence & Treatments \\
\hline \multirow{5}{*}{$\begin{array}{c}3-(1,1- \\
\text { dimethylethyl)- } \\
\text { phenol }\end{array}$} & \multirow{5}{*}{$\mathrm{C}_{10} \mathrm{H}_{16} \mathrm{O}$} & \multirow{5}{*}{33} & \multirow{5}{*}{26.455} & \multirow{5}{*}{1.80} & \multirow{5}{*}{88} & - & Control \\
\hline & & & & & & - & $\mathrm{nCeO} 2$ \\
\hline & & & & & & + & G-CeO ${ }_{2}$ \\
\hline & & & & & & + & $\mathrm{L}-\mathrm{CeO}_{2}$ \\
\hline & & & & & & + & $\mathrm{P}-\mathrm{CeO}_{2}$ \\
\hline \multirow{5}{*}{$\begin{array}{c}\text { 1-methyl- } \\
\text { naphthalene }\end{array}$} & \multirow{5}{*}{$\mathrm{C}_{11} \mathrm{H}_{10}$} & \multirow{5}{*}{34} & \multirow{5}{*}{26.759} & \multirow{5}{*}{2.04} & \multirow{5}{*}{88} & - & Control \\
\hline & & & & & & - & $\mathrm{nCeO} 2$ \\
\hline & & & & & & + & $\mathrm{G}-\mathrm{CeO}_{2}$ \\
\hline & & & & & & + & $\mathrm{L}-\mathrm{CeO}_{2}$ \\
\hline & & & & & & + & $\mathrm{P}-\mathrm{CeO}_{2}$ \\
\hline \multirow{5}{*}{ Undecanal } & \multirow{5}{*}{$\mathrm{C}_{11} \mathrm{H}_{22} \mathrm{O}$} & \multirow{5}{*}{35} & \multirow{5}{*}{26.945} & \multirow{5}{*}{1.20} & & - & Control \\
\hline & & & & & & - & $\mathrm{nCeO} 2$ \\
\hline & & & & & 93 & + & $\mathrm{G}-\mathrm{CeO}_{2}$ \\
\hline & & & & & & + & $\mathrm{L}-\mathrm{CeO}_{2}$ \\
\hline & & & & & & + & $\mathrm{P}-\mathrm{CeO}_{2}$ \\
\hline & & & & & & - & Control \\
\hline & & & & & & - & $\mathrm{nCeO} 2$ \\
\hline $\begin{array}{l}(E, E)-2,4- \\
\text { decadienal }\end{array}$ & $\mathrm{C}_{10} \mathrm{H}_{16} \mathrm{O}$ & 36 & 27.051 & 1.53 & 89 & + & $\mathrm{G}-\mathrm{CeO}_{2}$ \\
\hline & & & & & & + & $\mathrm{L}-\mathrm{CeO}_{2}$ \\
\hline & & & & & & + & $\mathrm{P}-\mathrm{CeO}_{2}$ \\
\hline & & & & & & - & Control \\
\hline & & & & & & - & $\mathrm{nCeO} 2$ \\
\hline $\begin{array}{l}1,2,3 \text {-piopanter } \\
\text { triacetate }\end{array}$ & $\mathrm{C}_{9} \mathrm{H}_{14} \mathrm{O}_{6}$ & 37 & 27.459 & 1.98 & 94 & + & G-CeO ${ }_{2}$ \\
\hline & & & & & & + & $\mathrm{L}-\mathrm{CeO}_{2}$ \\
\hline & & & & & & + & $\mathrm{P}-\mathrm{CeO}_{2}$ \\
\hline & & & & & & - & Control \\
\hline & & & & & & - & $\mathrm{nCeO} 2$ \\
\hline 2-dodecenal & $\mathrm{C}_{12} \mathrm{H}_{22} \mathrm{O}$ & 38 & 28.448 & 1.35 & 89 & + & $\mathrm{G}-\mathrm{CeO}_{2}$ \\
\hline & & & & & & + & $\mathrm{L}-\mathrm{CeO}_{2}$ \\
\hline & & & & & & + & $\mathrm{P}-\mathrm{CeO}_{2}$ \\
\hline & & & & & & - & Control \\
\hline $\begin{array}{c}1,2,3,4- \\
\text { tetrabydro-1 } 6-\end{array}$ & & & & & & - & $\mathrm{nCeO} 2$ \\
\hline $\begin{array}{l}\text { tetrahydro-1,1,6- } \\
\text { trimethyl- }\end{array}$ & $\mathrm{C}_{13} \mathrm{H}_{18}$ & 39 & 28.551 & 1.56 & 92 & + & $\mathrm{G}-\mathrm{CeO}_{2}$ \\
\hline & & & & & & + & $\mathrm{L}-\mathrm{CeO}_{2}$ \\
\hline & & & & & & + & $\mathrm{P}-\mathrm{CeO}_{2}$ \\
\hline & & & & & & + & Control \\
\hline 3-hydroxy-2,4,4- & & & & & & + & $\mathrm{nCeO} 2$ \\
\hline $\begin{array}{l}\text { methyl-. } \\
\text { mols }\end{array}$ & $\mathrm{C}_{12} \mathrm{H}_{24} \mathrm{O}_{3}$ & 40 & 28.848 & 1.38 & 91 & + & G-CeO ${ }_{2}$ \\
\hline propanoate & & & & & & + & $\mathrm{L}-\mathrm{CeO}_{2}$ \\
\hline & & & & & & + & $\mathrm{P}-\mathrm{CeO}_{2}$ \\
\hline & & & & & & - & Control \\
\hline & & & & & & - & $\mathrm{nCeO} 2$ \\
\hline 2-ethenyl- & $\mathrm{C}_{12} \mathrm{H}_{10}$ & 41 & 28.962 & 2.19 & 89 & + & $\mathrm{G}-\mathrm{CeO}_{2}$ \\
\hline & & & & & & + & $\mathrm{L}-\mathrm{CeO}_{2}$ \\
\hline & & & & & & + & $\mathrm{P}-\mathrm{CeO}_{2}$ \\
\hline & & & & & & - & Control \\
\hline & & & & & & - & $\mathrm{nCeO} 2$ \\
\hline Dodecanal & $\mathrm{C}_{12} \mathrm{H}_{24} \mathrm{O}$ & 42 & 29.646 & 1.23 & 97 & + & $\mathrm{G}-\mathrm{CeO}_{2}$ \\
\hline & & & & & & + & $\mathrm{L}-\mathrm{CeO}_{2}$ \\
\hline & & & & & & + & $\mathrm{P}-\mathrm{CeO}_{2}$ \\
\hline & & & & & & - & Control \\
\hline & & & & & & - & $\mathrm{nCeO} 2$ \\
\hline 1,6-dimethyl- & $\mathrm{C}_{12} \mathrm{H}_{12}$ & 43 & 29.759 & 1.98 & 92 & + & G-CeO ${ }_{2}$ \\
\hline & & & & & & + & $\mathrm{L}-\mathrm{CeO}_{2}$ \\
\hline & & & & & & + & $\mathrm{P}-\mathrm{CeO}_{2}$ \\
\hline
\end{tabular}




\begin{tabular}{|c|c|c|c|c|c|c|c|}
\hline Compounds & Formula & $\begin{array}{c}\text { Order } \\
\text { number }\end{array}$ & RT1 & RT2 & $\begin{array}{c}\text { Similarity } \\
(\%)\end{array}$ & Presence & Treatments \\
\hline \multirow{5}{*}{$\begin{array}{l}\text { 1,8-dimethyl- } \\
\text { naphthalene }\end{array}$} & \multirow{5}{*}{$\mathrm{C}_{12} \mathrm{H}_{12}$} & \multirow{5}{*}{44} & \multirow{5}{*}{30.160} & \multirow{5}{*}{2.07} & \multirow{5}{*}{81} & - & Control \\
\hline & & & & & & - & $\mathrm{nCeO} 2$ \\
\hline & & & & & & + & G-CeO ${ }_{2}$ \\
\hline & & & & & & + & $\mathrm{L}-\mathrm{CeO}_{2}$ \\
\hline & & & & & & + & $\mathrm{P}-\mathrm{CeO}_{2}$ \\
\hline \multirow{5}{*}{$\begin{array}{c}(1 R, 2 S, 7 S, 9 S)- \\
3,3,7-\text { trimethyl-8- } \\
\text { methylidenetricycl } \\
\left.\text { o[5.4.0.0 } 0^{2,9}\right] \text { undec } \\
\text { an } \\
\end{array}$} & \multirow{5}{*}{$\mathrm{C}_{15} \mathrm{H}_{24}$} & \multirow{5}{*}{45} & \multirow{5}{*}{30.347} & \multirow{5}{*}{1.26} & \multirow{5}{*}{91} & $\overline{-}$ & Control \\
\hline & & & & & & - & $\mathrm{nCeO} 2$ \\
\hline & & & & & & + & G- $\mathrm{CeO}_{2}$ \\
\hline & & & & & & + & $\mathrm{L}-\mathrm{CeO}_{2}$ \\
\hline & & & & & & - & $\mathrm{P}-\mathrm{CeO}_{2}$ \\
\hline \multirow{5}{*}{$\begin{array}{l}\text { 6,10-dimethyl- } \\
5,9 \text {-undecadien-2- } \\
\text { one }\end{array}$} & \multirow{5}{*}{$\mathrm{C}_{13} \mathrm{H}_{22} \mathrm{O}$} & \multirow{5}{*}{46} & \multirow{5}{*}{30.749} & \multirow{5}{*}{1.41} & & - & Control \\
\hline & & & & & & - & $\mathrm{nCeO} 2$ \\
\hline & & & & & 87 & + & $\mathrm{G}-\mathrm{CeO}_{2}$ \\
\hline & & & & & & + & $\mathrm{L}-\mathrm{CeO}_{2}$ \\
\hline & & & & & & + & $\mathrm{P}-\mathrm{CeO}_{2}$ \\
\hline & & & & & & - & Control \\
\hline 2,6-di-butyl-2,5- & & & & & & - & $\mathrm{nCeO} 2$ \\
\hline cyclohexadiene- & $\mathrm{C}_{14} \mathrm{H}_{20} \mathrm{O}_{2}$ & 47 & 31.250 & 1.44 & 82 & + & G-CeO ${ }_{2}$ \\
\hline & & & & & & + & $\mathrm{L}-\mathrm{CeO}_{2}$ \\
\hline & & & & & & + & $\mathrm{P}-\mathrm{CeO}_{2}$ \\
\hline & & & & & & - & Control \\
\hline 4-(2,6,6-trimethyl- & & & & & & - & $\mathrm{nCeO} 2$ \\
\hline 1-cyclohexen-1- & $\mathrm{C}_{13} \mathrm{H}_{20} \mathrm{O}$ & 48 & 31.753 & 1.62 & 91 & + & $\mathrm{G}-\mathrm{CeO}_{2}$ \\
\hline & & & & & & + & $\mathrm{L}-\mathrm{CeO}_{2}$ \\
\hline & & & & & & + & $\mathrm{P}-\mathrm{CeO}_{2}$ \\
\hline & & & & & & - & Control \\
\hline & & & & & & - & $\mathrm{nCeO} 2$ \\
\hline Tridecanal & $\mathrm{C}_{13} \mathrm{H}_{26} \mathrm{O}$ & 49 & 32.246 & 1.23 & 91 & + & $\mathrm{G}-\mathrm{CeO}_{2}$ \\
\hline & & & & & & + & $\mathrm{L}-\mathrm{CeO}_{2}$ \\
\hline & & & & & & + & $\mathrm{P}-\mathrm{CeO}_{2}$ \\
\hline & & & & & & - & Control \\
\hline 2,4-bis(1,1- & & & & & & - & $\mathrm{nCeO} 2$ \\
\hline dimethylethyl)- & $\mathrm{C}_{16} \mathrm{H}_{26} \mathrm{O}$ & 50 & 32.251 & 1.53 & 91 & + & $\mathrm{G}-\mathrm{CeO}_{2}$ \\
\hline & & & & & & + & $\mathrm{L}-\mathrm{CeO}_{2}$ \\
\hline & & & & & & + & $\mathrm{P}-\mathrm{CeO}_{2}$ \\
\hline & & & & & & - & Control \\
\hline & & & & & & - & $\mathrm{nCeO} 2$ \\
\hline $\begin{array}{c}O- \\
\text { hydroxybinhenyl }\end{array}$ & $\mathrm{C}_{12} \mathrm{H}_{10} \mathrm{O}$ & 51 & 32.269 & 2.58 & 95 & + & G-CeO ${ }_{2}$ \\
\hline & & & & & & + & $\mathrm{L}-\mathrm{CeO}_{2}$ \\
\hline & & & & & & + & $\mathrm{P}-\mathrm{CeO}_{2}$ \\
\hline & & & & & & - & Control \\
\hline $\begin{array}{l}\text { cis-hexahydro-8a- } \\
\text { methyl- }\end{array}$ & & & & & & - & $\mathrm{nCeO} 2$ \\
\hline $\begin{array}{l}\text { methyl-, } \\
1,8(2 \mathrm{H}, 5 \mathrm{H})-\end{array}$ & $\mathrm{C}_{11} \mathrm{H}_{16} \mathrm{O}_{2}$ & 52 & 32.359 & 1.98 & 81 & + & $\mathrm{G}-\mathrm{CeO}_{2}$ \\
\hline naphthalenedione & & & & & & + & $\mathrm{L}-\mathrm{CeO}_{2}$ \\
\hline & & & & & & + & $\mathrm{P}-\mathrm{CeO}_{2}$ \\
\hline & & & & & & - & Control \\
\hline $\begin{array}{l}\text { (R)- }-5,0,1,1 \mathrm{~A}- \\
\text { tetrahydro-4,4,7A- }\end{array}$ & & & & & & - & $\mathrm{nCeO} 2$ \\
\hline $\begin{array}{l}\text { terranyaro-4,4,/A- } \\
\text { trimethyl-2(4H)- }\end{array}$ & $\mathrm{C}_{11} \mathrm{H}_{16} \mathrm{O}_{2}$ & 53 & 32.471 & 2.70 & 89 & + & $\mathrm{G}-\mathrm{CeO}_{2}$ \\
\hline benzofuranone & & & & & & + & $\mathrm{L}-\mathrm{CeO}_{2}$ \\
\hline & & & & & & + & $\mathrm{P}-\mathrm{CeO}_{2}$ \\
\hline & & & & & & - & Control \\
\hline & & & & & & - & $\mathrm{nCeO} 2$ \\
\hline $2,6,10-$ & $\mathrm{C}_{15} \mathrm{H}_{26} \mathrm{O}$ & 54 & 32.650 & 1.44 & 84 & + & G-CeO ${ }_{2}$ \\
\hline dodecatrien-1-ol & & & & & & + & $\mathrm{L}-\mathrm{CeO}_{2}$ \\
\hline & & & & & & + & $\mathrm{P}-\mathrm{CeO}_{2}$ \\
\hline
\end{tabular}




\begin{tabular}{|c|c|c|c|c|c|c|c|}
\hline Compounds & Formula & $\begin{array}{c}\text { Order } \\
\text { number }\end{array}$ & RT1 & RT2 & $\begin{array}{c}\text { Similarity } \\
(\%)\end{array}$ & Presence & Treatments \\
\hline \multirow{5}{*}{$\begin{array}{l}\text { 2-methyl-1- } \\
\text { pentadecene }\end{array}$} & \multirow{5}{*}{$\mathrm{C}_{16} \mathrm{H}_{32}$} & \multirow{5}{*}{55} & \multirow{5}{*}{33.044} & \multirow{5}{*}{1.11} & \multirow{5}{*}{81} & - & Control \\
\hline & & & & & & - & $\mathrm{nCeO} 2$ \\
\hline & & & & & & + & G-CeO ${ }_{2}$ \\
\hline & & & & & & + & $\mathrm{L}-\mathrm{CeO}_{2}$ \\
\hline & & & & & & - & $\mathrm{P}-\mathrm{CeO}_{2}$ \\
\hline \multirow{5}{*}{$\begin{array}{c}\text { Diethyl-1,2- } \\
\text { benzenedicarboxy } \\
\text { late }\end{array}$} & \multirow{5}{*}{$\mathrm{C}_{12} \mathrm{H}_{14} \mathrm{O}_{4}$} & \multirow{5}{*}{56} & \multirow{5}{*}{33.767} & \multirow{5}{*}{2.46} & \multirow{5}{*}{89} & - & Control \\
\hline & & & & & & - & $\mathrm{nCeO} 2$ \\
\hline & & & & & & + & G-CeO ${ }_{2}$ \\
\hline & & & & & & + & $\mathrm{L}-\mathrm{CeO}_{2}$ \\
\hline & & & & & & + & $\mathrm{P}-\mathrm{CeO}_{2}$ \\
\hline \multirow{5}{*}{$\begin{array}{l}\text { Isobutyl-2,2,4- } \\
\text { trimethyl-3- } \\
\text { carboxyisopropyl- } \\
\text { pentanoate }\end{array}$} & \multirow{5}{*}{$\mathrm{C}_{16} \mathrm{H}_{30} \mathrm{O}_{4}$} & \multirow{5}{*}{57} & \multirow{5}{*}{34.447} & \multirow{5}{*}{1.26} & & - & Control \\
\hline & & & & & & - & $\mathrm{nCeO} 2$ \\
\hline & & & & & 90 & + & $\mathrm{G}-\mathrm{CeO}_{2}$ \\
\hline & & & & & & + & $\mathrm{L}-\mathrm{CeO}_{2}$ \\
\hline & & & & & & + & $\mathrm{P}-\mathrm{CeO}_{2}$ \\
\hline & & & & & & - & Control \\
\hline & & & & & & + & $\mathrm{nCeO} 2$ \\
\hline Tetradecanal & $\mathrm{C}_{14} \mathrm{H}_{28} \mathrm{O}$ & 58 & 34.647 & 1.26 & 93 & + & G-CeO ${ }_{2}$ \\
\hline & & & & & & + & $\mathrm{L}-\mathrm{CeO}_{2}$ \\
\hline & & & & & & + & $\mathrm{P}-\mathrm{CeO}_{2}$ \\
\hline & & & & & & - & Control \\
\hline & & & & & & - & $\mathrm{nCeO} 2$ \\
\hline $\begin{array}{l}\text { dodecanoate } \\
\text { dodiopyi }\end{array}$ & $\mathrm{C}_{15} \mathrm{H}_{30} \mathrm{O}_{2}$ & 59 & 35.045 & 1.14 & 88 & - & G-CeO ${ }_{2}$ \\
\hline & & & & & & + & $\mathrm{L}-\mathrm{CeO}_{2}$ \\
\hline & & & & & & + & $\mathrm{P}-\mathrm{CeO}_{2}$ \\
\hline & & & & & & - & Control \\
\hline Methyl-(3-oxo-2- & & & & & & - & $\mathrm{nCeO} 2$ \\
\hline pentyl-, & $\mathrm{C}_{13} \mathrm{H}_{22} \mathrm{O}_{3}$ & 60 & 35.357 & 1.89 & 89 & + & G-CeO ${ }_{2}$ \\
\hline & & & & & & + & $\mathrm{L}-\mathrm{CeO}_{2}$ \\
\hline & & & & & & + & $\mathrm{P}-\mathrm{CeO}_{2}$ \\
\hline & & & & & & - & Control \\
\hline & & & & & & - & $\mathrm{nCeO} 2$ \\
\hline n-hexyl salicylate & $\mathrm{C}_{13} \mathrm{H}_{18} \mathrm{O}_{3}$ & 61 & 36.154 & 1.71 & 84 & + & G-CeO ${ }_{2}$ \\
\hline & & & & & & + & $\mathrm{L}-\mathrm{CeO}_{2}$ \\
\hline & & & & & & - & $\mathrm{P}-\mathrm{CeO}_{2}$ \\
\hline & & & & & & - & Control \\
\hline & & & & & & - & $\mathrm{nCeO} 2$ \\
\hline 1-tetradecanol & $\mathrm{C}_{14} \mathrm{H}_{30} \mathrm{O}$ & 62 & 36.447 & 1.26 & 88 & + & G-CeO ${ }_{2}$ \\
\hline & & & & & & + & $\mathrm{L}-\mathrm{CeO}_{2}$ \\
\hline & & & & & & + & $\mathrm{P}-\mathrm{CeO}_{2}$ \\
\hline & & & & & & - & Control \\
\hline & & & & & & - & $\mathrm{nCeO} 2$ \\
\hline 13-tetradecenal & $\mathrm{C}_{14} \mathrm{H}_{26} \mathrm{O}$ & 63 & 36.549 & 1.38 & 88 & + & $\mathrm{G}-\mathrm{CeO}_{2}$ \\
\hline & & & & & & + & $\mathrm{L}-\mathrm{CeO}_{2}$ \\
\hline & & & & & & + & $\mathrm{P}-\mathrm{CeO}_{2}$ \\
\hline & & & & & & + & Control \\
\hline & & & & & & + & $\mathrm{nCeO} 2$ \\
\hline Pentadecanal & $\mathrm{C}_{15} \mathrm{H}_{30} \mathrm{O}$ & 64 & 36.947 & 1.29 & 93 & + & $\mathrm{G}-\mathrm{CeO}_{2}$ \\
\hline & & & & & & + & $\mathrm{L}-\mathrm{CeO}_{2}$ \\
\hline & & & & & & + & $\mathrm{P}-\mathrm{CeO}_{2}$ \\
\hline & & & & & & - & Control \\
\hline & & & & & & - & $\mathrm{nCeO} 2$ \\
\hline Triethyl- $O$ - & $\mathrm{C}_{14} \mathrm{H}_{22} \mathrm{O}_{8}$ & 65 & 37.259 & 2.04 & 87 & + & G-CeO ${ }_{2}$ \\
\hline & & & & & & + & $\mathrm{L}-\mathrm{CeO}_{2}$ \\
\hline & & & & & & - & $\mathrm{P}-\mathrm{CeO}_{2}$ \\
\hline
\end{tabular}




\begin{tabular}{|c|c|c|c|c|c|c|c|}
\hline Compounds & Formula & $\begin{array}{c}\text { Order } \\
\text { number }\end{array}$ & RT1 & RT2 & $\begin{array}{c}\text { Similarity } \\
(\%)\end{array}$ & Presence & Treatments \\
\hline \multirow{5}{*}{$\begin{array}{c}\text { (6S-cis)-5,6,7,7A- } \\
\text { tetrahydro-6- } \\
\text { hydroxy-4,4,7A- } \\
\text { trimethyl-, } 2(4 \mathrm{H})- \\
\text { benzofuranone }\end{array}$} & \multirow{5}{*}{$\mathrm{C}_{11} \mathrm{H}_{16} \mathrm{O}_{3}$} & \multirow{5}{*}{66} & \multirow{5}{*}{37.486} & \multirow{5}{*}{3.63} & \multirow{5}{*}{85} & - & Control \\
\hline & & & & & & - & $\mathrm{nCeO} 2$ \\
\hline & & & & & & + & $\mathrm{G}-\mathrm{CeO}_{2}$ \\
\hline & & & & & & + & $\mathrm{L}-\mathrm{CeO}_{2}$ \\
\hline & & & & & & + & $\mathrm{P}-\mathrm{CeO}_{2}$ \\
\hline \multirow{5}{*}{ Docosanoic acid } & \multirow{5}{*}{$\mathrm{C}_{22} \mathrm{H}_{44} \mathrm{O}_{2}$} & \multirow{5}{*}{67} & \multirow{5}{*}{37.849} & \multirow{5}{*}{1.35} & \multirow{5}{*}{82} & - & Control \\
\hline & & & & & & - & $\mathrm{nCeO} 2$ \\
\hline & & & & & & - & $\mathrm{G}-\mathrm{CeO}_{2}$ \\
\hline & & & & & & + & $\mathrm{L}-\mathrm{CeO}_{2}$ \\
\hline & & & & & & + & $\mathrm{P}-\mathrm{CeO}_{2}$ \\
\hline \multirow{5}{*}{$\begin{array}{c}\text { 2-methyl-2- } \\
\text { phenyl-tridecane }\end{array}$} & \multirow{5}{*}{$\mathrm{C}_{20} \mathrm{H}_{34}$} & \multirow{5}{*}{68} & \multirow{5}{*}{38.661} & \multirow{5}{*}{2.10} & & - & Control \\
\hline & & & & & & - & $\mathrm{nCeO} 2$ \\
\hline & & & & & 80 & + & $\mathrm{G}-\mathrm{CeO}_{2}$ \\
\hline & & & & & & + & $\mathrm{L}-\mathrm{CeO}_{2}$ \\
\hline & & & & & & + & $\mathrm{P}-\mathrm{CeO}_{2}$ \\
\hline & & & & & & - & Control \\
\hline & & & & & & - & $\mathrm{nCeO} 2$ \\
\hline 6,10,14-trimethyl- & $\mathrm{C}_{18} \mathrm{H}_{36} \mathrm{O}$ & 69 & 38.748 & 1.32 & 84 & - & G-CeO ${ }_{2}$ \\
\hline & & & & & & + & $\mathrm{L}-\mathrm{CeO}_{2}$ \\
\hline & & & & & & + & $\mathrm{P}-\mathrm{CeO}_{2}$ \\
\hline & & & & & & + & Control \\
\hline & & & & & & - & $\mathrm{nCeO} 2$ \\
\hline Hexadecanal & $\mathrm{C}_{16} \mathrm{H}_{32} \mathrm{O}$ & 70 & 39.048 & 1.32 & 90 & - & G-CeO ${ }_{2}$ \\
\hline & & & & & & + & $\mathrm{L}-\mathrm{CeO}_{2}$ \\
\hline & & & & & & + & $\mathrm{P}-\mathrm{CeO}_{2}$ \\
\hline & & & & & & - & Control \\
\hline & & & & & & - & $\mathrm{nCeO} 2$ \\
\hline$N, N$-diethyl-1- & $\mathrm{C}_{14} \mathrm{H}_{31} \mathrm{~N}$ & 71 & 39.450 & 1.44 & 89 & + & $\mathrm{G}-\mathrm{CeO}_{2}$ \\
\hline & & & & & & + & $\mathrm{L}-\mathrm{CeO}_{2}$ \\
\hline & & & & & & + & $\mathrm{P}-\mathrm{CeO}_{2}$ \\
\hline & & & & & & - & Control \\
\hline methylpropyl)- & & & & & & - & $\mathrm{nCeO} 2$ \\
\hline & $\mathrm{C}_{16} \mathrm{H}_{22} \mathrm{O}_{4}$ & 72 & 39.762 & 2.16 & 94 & + & G-CeO ${ }_{2}$ \\
\hline benzenedicarboxy & & & & & & + & $\mathrm{L}-\mathrm{CeO}_{2}$ \\
\hline & & & & & & + & $\mathrm{P}-\mathrm{CeO}_{2}$ \\
\hline & & & & & & - & Control \\
\hline & & & & & & - & $\mathrm{nCeO} 2$ \\
\hline Heptyl benzoate & $\mathrm{C}_{14} \mathrm{H}_{20} \mathrm{O}_{2}$ & 73 & 39.955 & 1.77 & 82 & + & G-CeO ${ }_{2}$ \\
\hline & & & & & & + & $\mathrm{L}-\mathrm{CeO}_{2}$ \\
\hline & & & & & & + & $\mathrm{P}-\mathrm{CeO}_{2}$ \\
\hline & & & & & & - & Control \\
\hline & & & & & & - & $\mathrm{nCeO} 2$ \\
\hline tetramethyl-1- & $\mathrm{C}_{20} \mathrm{H}_{40} \mathrm{O}$ & 74 & 41.846 & 1.2 & 90 & - & $\mathrm{G}-\mathrm{CeO}_{2}$ \\
\hline hexadecen-3-ol & & & & & & - & $\mathrm{L}-\mathrm{CeO}_{2}$ \\
\hline & & & & & & + & $\mathrm{P}-\mathrm{CeO}_{2}$ \\
\hline & & & & & & - & Control \\
\hline & & & & & & - & $\mathrm{nCeO} 2$ \\
\hline$n$-hexadecanoic & $\mathrm{C}_{16} \mathrm{H}_{32} \mathrm{O}_{2}$ & 75 & 41.849 & 1.41 & 92 & - & G-CeO ${ }_{2}$ \\
\hline & & & & & & + & $\mathrm{L}-\mathrm{CeO}_{2}$ \\
\hline & & & & & & + & $\mathrm{P}-\mathrm{CeO}_{2}$ \\
\hline & & & & & & - & Control \\
\hline & & & & & & - & $\mathrm{nCeO} 2$ \\
\hline $\begin{array}{c}Z, E-3,13- \\
\text { gctadecadien-1-ol }\end{array}$ & $\mathrm{C}_{18} \mathrm{H}_{34} \mathrm{O}$ & 76 & 42.452 & 1.56 & 84 & - & $\mathrm{G}-\mathrm{CeO}_{2}$ \\
\hline & & & & & & - & $\mathrm{L}-\mathrm{CeO}_{2}$ \\
\hline & & & & & & + & $\mathrm{P}-\mathrm{CeO}_{2}$ \\
\hline
\end{tabular}




\begin{tabular}{|c|c|c|c|c|c|c|c|}
\hline Compounds & Formula & $\begin{array}{c}\text { Order } \\
\text { number }\end{array}$ & RT1 & RT2 & $\begin{array}{c}\text { Similarity } \\
(\%)\end{array}$ & Presence & Treatments \\
\hline \multirow{5}{*}{ Heptadecanal } & \multirow{5}{*}{$\mathrm{C}_{17} \mathrm{H}_{34} \mathrm{O}$} & \multirow{5}{*}{77} & \multirow{5}{*}{43.049} & \multirow{5}{*}{1.38} & \multirow{5}{*}{93} & + & Control \\
\hline & & & & & & - & $\mathrm{nCeO} 2$ \\
\hline & & & & & & + & G-CeO ${ }_{2}$ \\
\hline & & & & & & + & $\mathrm{L}-\mathrm{CeO}_{2}$ \\
\hline & & & & & & + & $\mathrm{P}-\mathrm{CeO}_{2}$ \\
\hline
\end{tabular}

Supplementary Table S3. Nonlinear principal component analysis; Component Loadings for wheat and pea samples

\begin{tabular}{lcc}
\hline & \multicolumn{2}{c}{ Wheat } \\
\hline & \multicolumn{2}{c}{ Dimension } \\
\hline V2 & 0.956 & -0.245 \\
V11 & 0.956 & -0.245 \\
V14 & 0.956 & -0.245 \\
V15 & 0.956 & -0.245 \\
V16 & 0.956 & -0.245 \\
V27 & 0.956 & -0.245 \\
V39 & 0.956 & -0.245 \\
V40 & 0.956 & -0.245 \\
V42 & 0.956 & -0.245 \\
V58 & 0.956 & -0.245 \\
V59 & 0.956 & -0.245 \\
V61 & 0.956 & -0.245 \\
V62 & 0.956 & -0.245 \\
V72 & 0.956 & -0.245 \\
V73 & 0.956 & -0.245 \\
V95 & 0.956 & -0.245 \\
\hline V3 & 0.811 & 0.409 \\
V10 & 0.811 & 0.409 \\
V12 & 0.811 & 0.409 \\
V17 & 0.811 & 0.409 \\
V18 & 0.811 & 0.409 \\
V24 & 0.811 & 0.409 \\
V33 & 0.811 & 0.409 \\
V35 & 0.811 & 0.409 \\
V105 & 0.811 & 0.409 \\
\hline V8 & 0.198 & -0.958 \\
V29 & 0.198 & -0.958 \\
V36 & 0.198 & -0.958 \\
\hline V21 & 0.540 & -0.052 \\
V23 & 0.540 & -0.052 \\
V67 & 0.540 & -0.052 \\
\hline & &
\end{tabular}

\begin{tabular}{lll}
\hline \multicolumn{3}{l}{ Pea } \\
\hline & 1 & 2 \\
\hline V3 & 0.401 & 0.812 \\
V74 & 0.401 & 0.812 \\
V76 & 0.401 & 0.812 \\
\hline V1 & 0.719 & 0.588 \\
V5 & 0.719 & 0.588 \\
V8 & 0.719 & 0.588 \\
V10 & 0.719 & 0.588 \\
V12 & 0.719 & 0.588 \\
V28 & 0.719 & 0.588 \\
V59 & 0.719 & 0.588 \\
V67 & 0.719 & 0.588 \\
V69 & 0.719 & 0.588 \\
V75 & 0.719 & 0.588 \\
\hline V19 & 0.668 & -0.696 \\
V20 & 0.668 & -0.696 \\
V21 & 0.668 & -0.696 \\
V25 & 0.668 & -0.696 \\
V45 & 0.668 & -0.696 \\
V55 & 0.668 & -0.696 \\
V61 & 0.668 & -0.696 \\
V65 & 0.668 & -0.696 \\
\hline V22 & 0.626 & 0.021 \\
V30 & 0.626 & 0.021 \\
V77 & 0.626 & 0.021 \\
\hline V4 & 0.996 & -0.033 \\
V6 & 0.996 & -0.033 \\
V7 & 0.996 & -0.033 \\
V9 & 0.996 & -0.033 \\
V11 & 0.996 & -0.033 \\
V13 & 0.996 & -0.033 \\
V14 & 0.996 & -0.033
\end{tabular}




\begin{tabular}{lrr}
\hline V32 & 0.510 & -0.662 \\
V47 & 0.510 & -0.662 \\
\hline V30 & 0.583 & 0.757 \\
V37 & 0.583 & 0.757 \\
V38 & 0.583 & 0.757 \\
V43 & 0.583 & 0.757 \\
V45 & 0.583 & 0.757 \\
V108 & 0.583 & 0.757 \\
\hline V41 & -0.331 & -0.565 \\
V78 & -0.327 & -0.548 \\
\hline V22 & -0.198 & 0.958 \\
V20 & -0.037 & -0.746 \\
V5 & -0.240 & 0.148 \\
V53 & 0.279 & -0.426 \\
V75 & 0.553 & 0.148 \\
V79 & 0.198 & -0.958 \\
V84 & 0.240 & -0.148 \\
V87 & -0.944 & 0.273 \\
V88 & -0.956 & 0.245 \\
\hline
\end{tabular}

\begin{tabular}{|c|c|c|}
\hline V15 & 0.996 & -0.033 \\
\hline V16 & 0.996 & -0.033 \\
\hline V17 & 0.996 & -0.033 \\
\hline V18 & 0.996 & -0.033 \\
\hline V23 & 0.996 & -0.033 \\
\hline V24 & 0.996 & -0.033 \\
\hline V26 & 0.996 & -0.033 \\
\hline V27 & 0.996 & -0.033 \\
\hline V29 & 0.996 & -0.033 \\
\hline V31 & 0.996 & -0.033 \\
\hline V32 & 0.996 & -0.033 \\
\hline V33 & 0.996 & -0.033 \\
\hline V34 & 0.996 & -0.033 \\
\hline V35 & 0.996 & -0.033 \\
\hline V36 & 0.996 & -0.033 \\
\hline V37 & 0.996 & -0.033 \\
\hline V38 & 0.996 & -0.033 \\
\hline V39 & 0.996 & -0.033 \\
\hline V41 & 0.996 & -0.033 \\
\hline V42 & 0.996 & -0.033 \\
\hline V43 & 0.996 & -0.033 \\
\hline V44 & 0.996 & -0.033 \\
\hline V46 & 0.996 & -0.033 \\
\hline V47 & 0.996 & -0.033 \\
\hline V48 & 0.996 & -0.033 \\
\hline V49 & 0.996 & -0.033 \\
\hline V50 & 0.996 & -0.033 \\
\hline V51 & 0.996 & -0.033 \\
\hline V52 & 0.996 & -0.033 \\
\hline V53 & 0.996 & -0.033 \\
\hline V54 & 0.996 & -0.033 \\
\hline V56 & 0.996 & -0.033 \\
\hline V57 & 0.996 & -0.033 \\
\hline V60 & 0.996 & -0.033 \\
\hline V62 & 0.996 & -0.033 \\
\hline V63 & 0.996 & -0.033 \\
\hline V66 & 0.996 & -0.033 \\
\hline V68 & 0.996 & -0.033 \\
\hline V71 & 0.996 & -0.033 \\
\hline V72 & 0.996 & -0.033 \\
\hline V73 & 0.996 & -0.033 \\
\hline V2 & 0.479 & -0.092 \\
\hline $\mathrm{V}$ & 0.594 & -0.061 \\
\hline
\end{tabular}




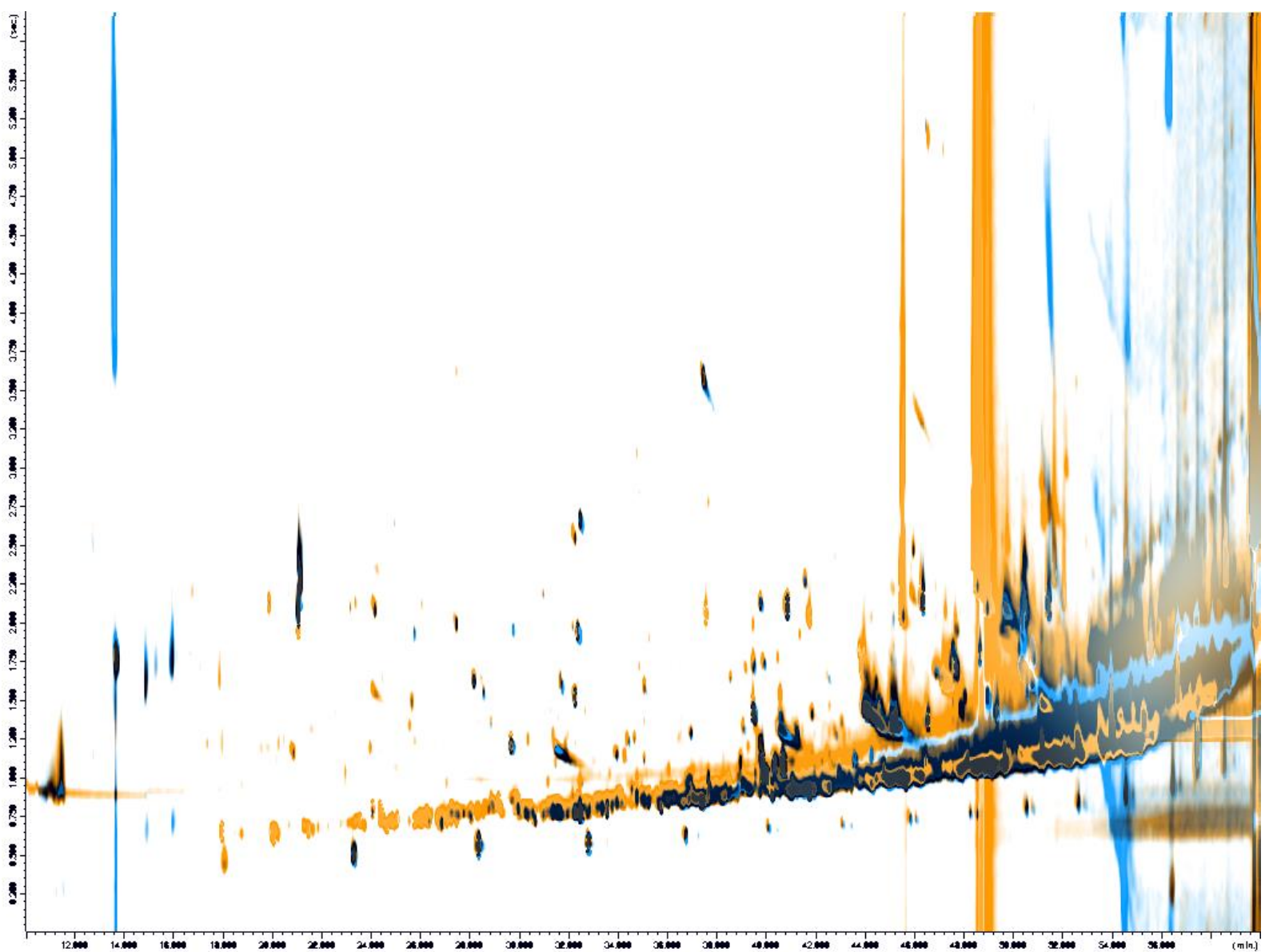

Supplementary Figure S1. Pair-wise image warping illustrated for wheat control and $\mathrm{nCeO}_{2}$ treated sample by using a dual-channel image (one image is false-colored in orange, the second one in blue) (The reader is referred to the Web version of the article for the interpretation of the references to color in this figure legend). 


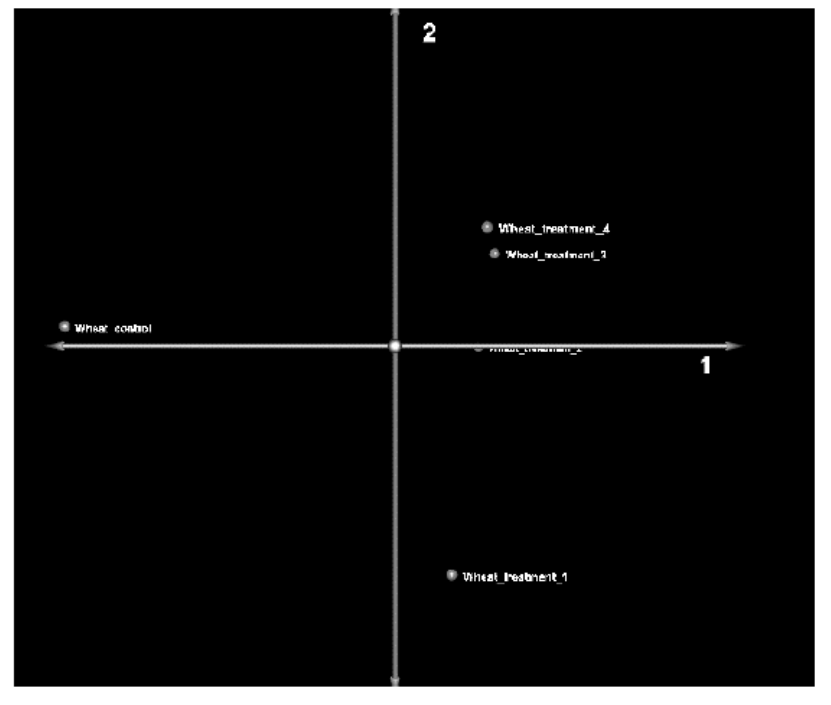

Wheat

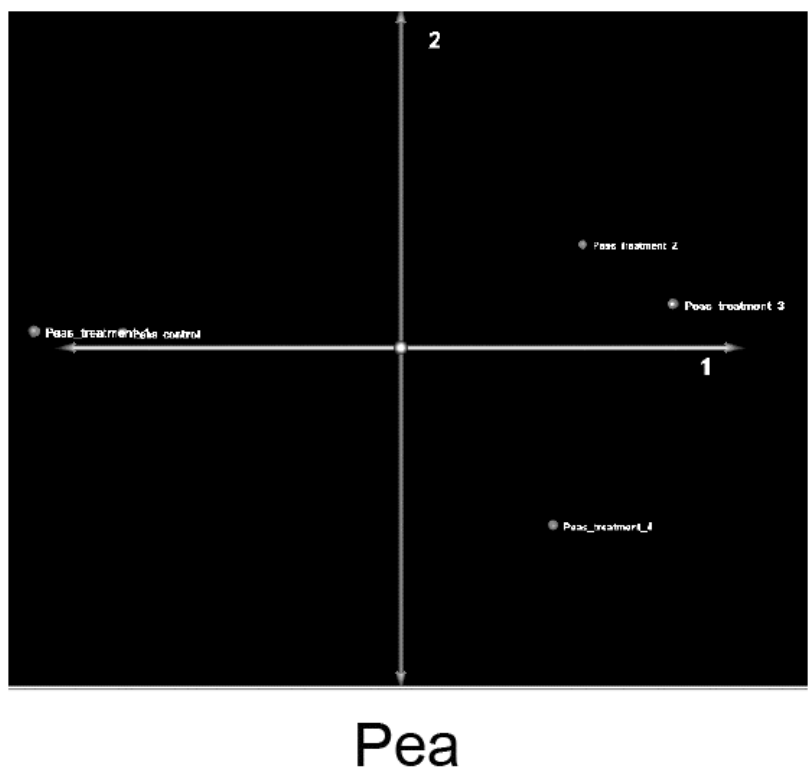

Pea

Supplementary Figure S2. Score plot of a linear principal component model for wheat, i.e. pea samples (treatment 1: $\mathrm{CeO}_{2}$, treatment 2: $\mathrm{G}-\mathrm{CeO}_{2}$, treatment 3: $\mathrm{L}-\mathrm{CeO}_{2}$, and treatment 4: $\left.\mathrm{P}-\mathrm{CeO}_{2}\right)$. 